\title{
Characterizing Crystalline Volcanic Rock (CVR) Deposits from Galiano Island, B.C., Canada: Implications for Lithic Material Procurement at the Dionisio Point Locality
}

\author{
Adam N. Rorabaugh ${ }^{1}$, Nichole S. Davenport ${ }^{2}$, and Colin Grier $^{3}$ \\ 1adam_rorabaugh@wsu.edu \\ 2nbettencourt@wsu.edu \\ ${ }^{3}$ cgrier@wsu.edu \\ Department of Anthropology, Washington State University \\ PO Box 644910, Pullman WA 99164-4910
}

KEY WORDS

Northwest Coast, Crystalline Volcanic Rock, WDXRF, Lithic Procurement, Toolstone Quality

\begin{abstract}
We define the elemental signatures of crystalline volcanic rock (CVR) found within the Dionisio Point archaeological locality on Galiano Island in coastal southwestern British Columbia. CVR is the dominant toolstone found in archaeological assemblages at Dionisio Point and many other sites in the region. The quality of these materials for chipped stone tool manufacture and use is dependent in part on the silica content, which is highly variable. Basalt is considered a poor quality toolstone due to its low silica content of $45-55 \%$, while andesite, dacite, and rhyolite are considered higher quality materials with silica content above 55\%. Elemental composition analyses were performed using WDXRF in the WSU Geoanalytical Lab to determine whether local secondary CVR deposits had high silica content. Results show that low and high quality CVR cobbles were locally available to the precontact communities of the Dionisio Point locality, with basalt being the most abundant.
\end{abstract}




\section{INTRODUCTION}

The pre-contact Coast Salish of the Salish Sea region of northwest Washington and southwest British Columbia have long held significance in literature theorizing sedentary foragers and small-scale complex societies (e.g., Ames, 1994, 1995, 2010; Angelbeck and Grier 2012; Kim and Grier, 2006; Grier 2014; Moss, 2011) (Figure 1). However, limited attention has been directed toward examining practices such as the acquisition and consumption of toolstone. This is partly due to the widespread use of bone, antler, and shell for tool manufacture, and their widespread preservation in the archaeological record (Stein, 1992; Stewart, 1996). However, formal and expedient chipped stone lithic technologies remained in use for a wide range of activities including hunting (terrestrial and marine), fishing, plant processing, and woodworking (Ames, 2009; Kornbacher, 1989; Stewart, 1996), and provide an important window into the organizational complexity of these practices.

The materials primarily utilized for chipped stone technologies in the Salish Sea have been referred to as basalts (Bakewell, 1996, 2005; Kornbacher, 1989, 1992; Kwarsick, 2010; Morgan, 1999; Wessen, 1993), crystalline volcanic rock (CVR) (Close, 2006), and fine-grained volcanic rock (FGV) (Taylor, 2012). All of these labels refer to the same general class of igneous materials and will be referred to as crystalline volcanic rock (CVR) in this study. As specific types of CVR such as basalts and dacites typically cannot be distinguished without elemental analyses, variation in the quality of these toolstones has been underexplored in the Northwest Coast literature. 


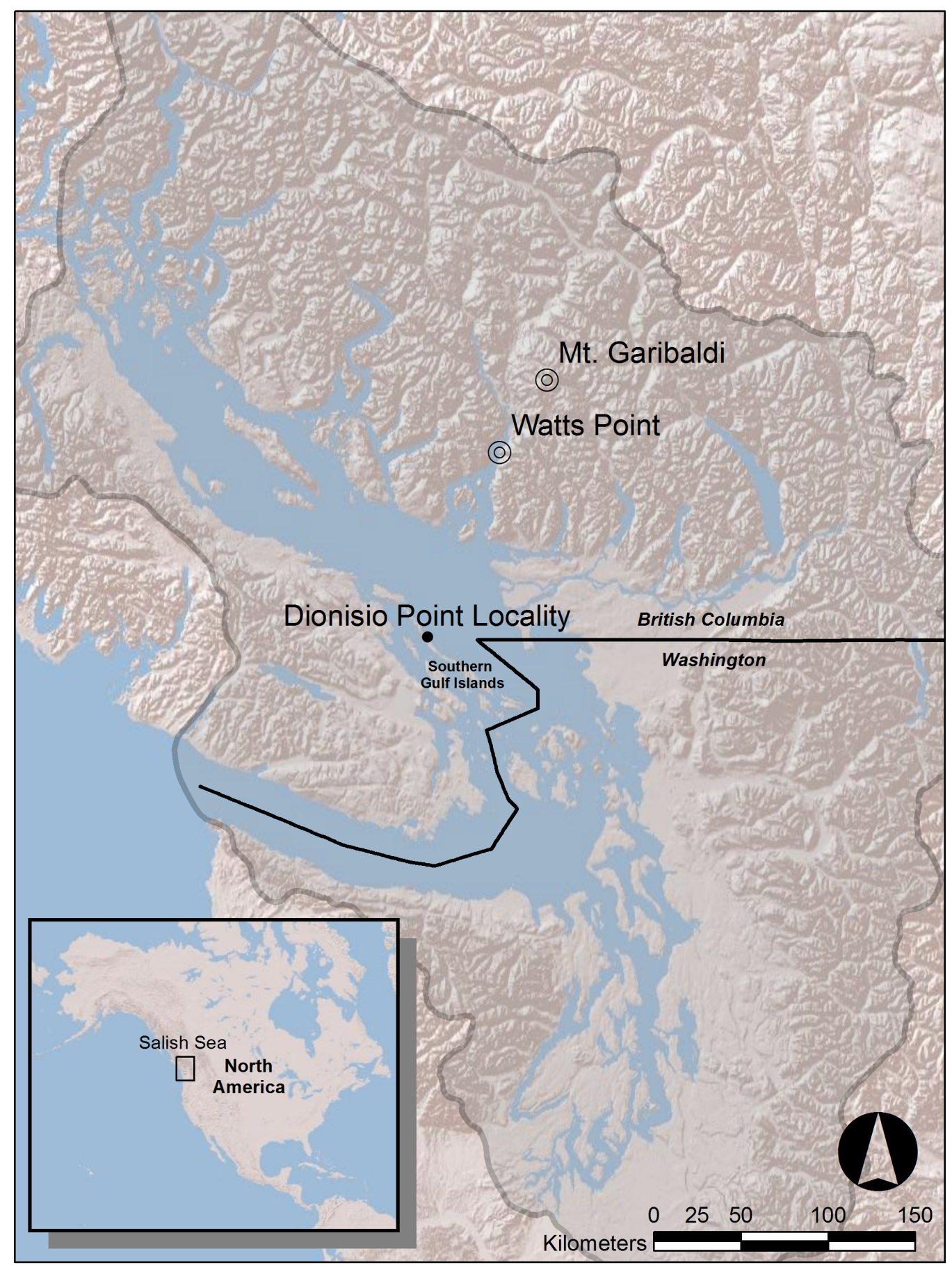

Figure 1 The location of the Dionisio Point locality and referenced lithic sources in the Salish Sea. 
The quality of CVR has been argued to be poor, which some suggest is why ground stone (slate, schist), bone, and antler technologies were so widely utilized (Ames, 2009; Graesch, 2007). Compared to obsidian or chert, CVR is more difficult to initially reduce, but it does keep a better edge and requires less retouch during use (Terry et al., 2009: 265; William Andrefsky Jr., personal communication, 2013). Despite the apparent diversification of materials used for tools manufacture through time (Ames, 2009), throughout the Holocene chipped stone technologies formed a key part of the tool kit and lifeways of the Coast Salish. Some have suggested that individuals traveled considerable distances to acquire higher quality CVR (Bakewell, 2005; Close, 2006). 
Sourcing studies have been employed to examine questions of regional exchange in the Salish Sea (Bakewell, 2005; Close, 2006; Kwarsick, 2010; Morgan et al., 1999; Reimer, 2011; Sobel, 2006; Taylor, 2012). Here we target the study of one major precontact village location, Dionisio Point, to consider tool acquisition strategies from the vantage point of an intensive habitation location with a large resource catchment zone (Grier 2003). We characterize the composition of locally available toolstone, lithic debitage, and formed chipped stone tools from this village locality to provide a focused study with regional implications, and which furthers our understanding of the organizational complexity of lithic procurement on the Northwest Coast.

To that end, we have sampled and characterized toolstone collected from beaches within the Dionisio Point locality and debitage and formed tools recovered from DgRv-6, a Late Period plankhouse and associated shell midden next to the beaches. The two questions we address are: 1) was there high quality toolstone available within the Dionisio Point Locality? and 2) is there evidence of selection for higher quality materials? If both are the case, long distance toolstone acquisition is unnecessary.

We begin this paper with a brief regional background and a review of past toolstone procurement studies in the Salish Sea, then provide a brief discussion of the Dionisio Point locality and its context in Coast Salish archaeology. Following this we discuss our sampling strategy for natural (beach and glacial till-derived) cobbles and cultural (debitage and formed tools) lithic materials at Dionisio Point. We then present the results of our Wavelength Dispersive X-ray Fluorescence Analysis (WDXRF) analysis. We conclude that high quality toolstones were readily available in secondary glacial till deposits at the Dionisio Point locality, and that there is evidence for the selection of higher quality toolstone. Finally, we discuss trace elements that can be used to distinguish between low and high quality CVR toolstones using non-destructive XRF analyses in future studies.

\subsection{Study Area}

The focus of our study is the Dionisio Point locality in the southern Gulf Islands of coastal British Columbia. The 
southern Gulf Islands are located in the archaeologically-important Salish Sea region of the Northwest Coast culture area (Figure 1). In terms of its natural, ethnographic and archaeological characteristics, the Salish Sea is a unique and distinct region of the Northwest Coast (Mitchell, 1971a).

At contact with Europeans, the southern Gulf Islands were inhabited by Hul'q'umi'num and Straits-speaking Coast Salish peoples. These First Nations were complex hunter-gatherer-fishers that lived in seasonally permanent villages. Their economies emphasized salmon procurement and storage but also gathered a wide range of marine and terrestrial resources (Bilton, 2014; Duer and Turner, 2005; Grier, 2006, 2014; McKechnie, 2014; see papers in Moss, 2011). While centered on the southern Gulf Islands and southeastern Vancouver Island, they maintained close historical ties with other Coast Salish speaking communities on the Fraser River and elsewhere (Grier, 2003; Mitchell, 1971a). Resources documented at the Dionisio Point locality were acquired from throughout the Salish Sea (Grier 2001, 2003).

This study focuses on the last 1500 years of Coast Salish prehistory, which includes the Marpole Period (2400-1000 BP) and Late Period (1000 BP-Contact). Marpole lifeways have received significant attention in the literature, as it is the first period on the southern BC coast with substantial large cedar plankhouse villages (Burley, 1980; Matson and Coupland, 1994; Thom, 1995). Archaeologists have long hypothesized significant connections between the development of large houses and villages on the Northwest Coast and the emergence of new forms of social inequality and, germane to our current study, formalized regional interaction networks (Ames, 1995: 181; Ames and Maschner, 1999; Beattie, 1981; Burley, 1980; Burley and Knusel, 1989; Lepofsky et al., 2009; Moss, 2011; Schaepe, 2009).

Importantly, the Marpole and subsequent Late Period are characterized by formalized regional exchange networks of lithic materials, both raw and finished (Grier, 2003). Exotic toolstone (e.g., nephrite, obsidian) moving over large distances has been interpreted as reflecting prestige exchange among elites and, more broadly, that exotic toolstone 
was a highly valued commodity. This has, as we discuss below, influenced how CVR distributions have been viewed. In this study, we provide an alternative to regional procurement hypotheses for CVR toolstone, arguing that, despite evidence for trade in exotic materials, locally available toolstone can account for the materials observed in archaeological assemblages from the Dionisio Point locality.

\subsection{Toolstone Procurement Studies in the Salish Sea}

Considerable debate has emerged regarding the locations where tool stone was procured in the Salish Sea region (Bakewell, 2005; Close, 2006; Kwarsick, 2010; Morgan, 1999; Reimer, 2011; Sobel, 2006; Taylor, 2012; Taylor and Skinner, 2010). Studies of Northwest Coast toolstone have emphasized obsidian sourcing (Carlson, 1994; Reimer, 2011; Sobel, 2006) and nephrite (Darwent, 1996; Morin, 2012), both of which have wide distributions derived from limited and localized sources. Toolstone obsidian recovered from archaeological sites in the Salish Sea predominantly comes from Mt. Garibaldi, and two sources on the central coast (Kingcome Glacier [Central Coast A] and Central Coast B) (Carlson, 1994; Reimer, 2011; Rorabaugh and McNabb, 2014; Skinner, 2009). Nephrite found throughout the Salish Sea comes from interior areas up the Fraser River several hundred kilometers inland. Given their wide distribution, these materials have been used primarily to examine the emergence of long distance exchange systems.

Despite the emphasis on obsidian and nephrite studies, CVR was a key resource for the manufacture of lithic technologies for pre-contact Coast Salish communities. Several studies exploring its sources and distribution have appeared (e.g., Bakewell, 2005; Kwarsick, 2010; Taylor, 2012; Taylor and Skinner, 2010). CVR, which include basalt, andesite, dacite, and rhyolite, comprise the majority of toolstone seen in many archaeological assemblages. A key aspect of the suitability of these materials for tool manufacture and use is their silica content, which is highly variable. Basalt is considered a poor quality toolstone due to its silica content of $45-55 \%$, while andesite, dacite, and rhyolite are considered higher quality materials with silica contents above 55\% (Le Maitre et al., 2002). 
Some of the highest quality CVR used in the Salish Sea has been identified as dacite from the Watts Point source in the Coast Range of British Columbia (Figure 1). Petrographic and elemental sourcing of Watts Point dacite has led to far reaching conclusions regarding toolstone procurement, with Bakewell (2005), Stein (2000), and Close (2006) proposing that dacite cobbles were procured directly from Watts Point in British Columbia and transported by canoe throughout the region. Recent analyses suggest that although Watts Point materials were widely distributed in the Salish Sea, this is likely the result of glacial movement and not human transport (Kwarsick, 2010; Taylor, 2012). CVRs may have been primarily collected from local contexts, as argued in a recent study by Taylor (2012) on San Juan Island. Several researchers have also noted CVR suitable for toolstone is present in local secondary beach deposits near archaeological sites (Conca, 2000; Herbel et al., 2001; Kenady et al., 1973; King, 1950; Kornbacher, 1992; Schalk, 1988; Wessen, 1993). If dacite is readily available from local secondary deposits, high quality CVRs may not serve as a strong indicator of regional exchange, as argued by Close (2006).

\subsection{The Dionisio Point Locality}

The Dionisio Point locality is situated on the northern tip of Galiano Island in the southern Gulf Island group. Galiano Island is part of the sedimentary formations of the Nanaimo Group, which consists of alternating beds of conglomerates, sandstones, and shale that date to the Upper Cretaceous (Green et al., 1989). Like several other of the southern Gulf Islands, Galiano Island is long and narrow, and reflects the northwest-southeast aspect of its underlying sedimentary rock (Johnstone, 2006). The sandstone and shale substrate is the parent material for most of the regolith, and served as toolstone for the local ground stone industry (Grier, 2001).

Volcanic rock is absent, except in sediments that contain transported material. The sediments of Galiano Island are the result of the Fraser Glaciation. Melting and retreating glaciers deposited glacial tills, which have been eroded, reworked and redeposited over the Holocene. These glacial tills form the parent materials for Gulf Island soils (Eis and Craigdallie, 1980; Green et al., 1989). Cobbles contained in these glacial deposits are present in modern beaches and in erosion exposures. Whether these cobbles were used for toolstone in the chipped stone industry is the focus of 
our study.

Post glacial sea level change in the southern Gulf Islands was more gradual than punctuated, Following rapid changes in the late Pleistocene and early Holocene, over the past 5,000 years sea levels have risen between 0.8-1.5m (Grier et al., 2009; Clague et al., 1982; Clague and James, 2002; Fedje et al., 2009; Mitchell, 1971b). Overall the environment at the Dionisio Point locality during its prehistoric occupation appears to be broadly similar to that of recent times (Grier, 2001).

Archaeologically, the Dionisio Point locality includes two major sites: the Dionisio Point site (DgRv-3), a Marpole Period Coast Salish village occupied between 1500 and 1300 cal BP (Grier, 2001, 2006) and DgRv-6, a Late Period plank house and associated midden inhabited from 1000 to 600 cal BP (Grier and McLay, 2007). DgRv-6 also contains a Marpole Period midden component along the shores of Parry Lagoon (Figure 2).

The earliest excavations at the Dionisio Point locality were by Mitchell at the Dionisio Point site (DgRv-3) in 1964 as part of his analysis of the Gulf of Georgia regional sequence (Mitchell, 1971a). Mitchell's excavations included several test units, and analyses emphasized the lithic assemblage (1971b). The site was revisited during 1997 and 1998 for the Dionisio Point Household Archaeology Project (DPHAP) by Grier (2001, 2003, 2006). These excavations yielded a relatively large artifact and faunal assemblage. Since then, additional excavations have occurred at the DgRv-3 village and the Late Period structure (House 1) and adjacent Parry Lagoon midden at DgRv-6. These projects, completed over a span of more than 50 years, have documented two important, substantial and well-preserved village occupations that extended over a millennia (Grier 2014). As such, Dionisio Point provides an ideal archaeological locality 
for examining regional and local patterns of exchange and resource acquisition. 


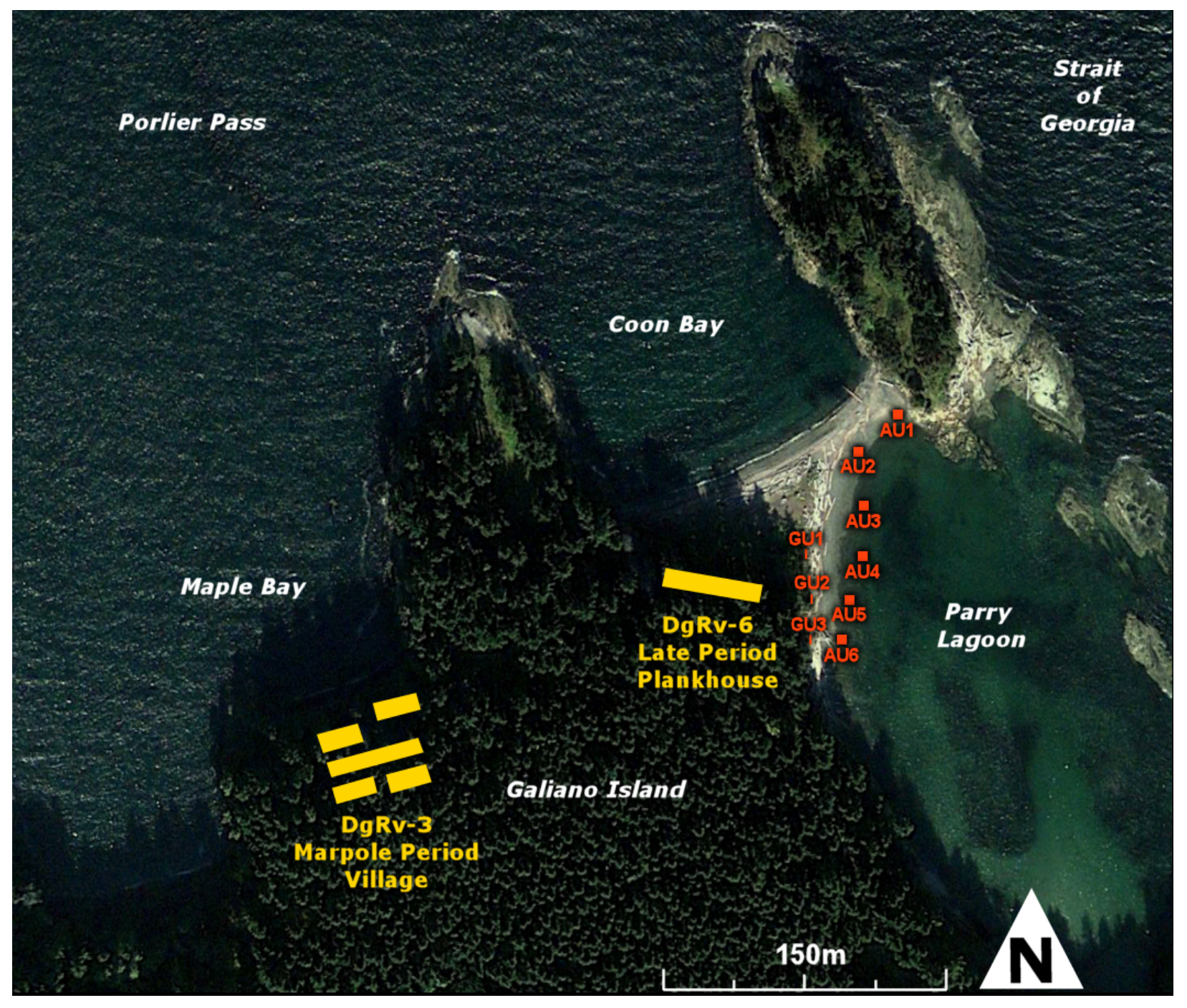

Figure 2 Parry's Lagoon natural control sampling locations. Note samples were collected at low tide, image shows high tide. (Base image from Google Earth). 


\section{MATERIALS AND METHODS}

Lithic materials recovered from the house deposits and Parry Lagoon midden at DgRv-6 form the cultural samples in this study (DGRV6), along with several lithic artifacts surface collected from the beaches along Coon Bay and Parry Lagoon (DBF). Natural samples were collected from the beach surface (BEA) and a wave cut bank (WCB) at Parry Lagoon. Samples were assigned to three groups for comparison: natural controls (BEA, WCB), beach finds (DBF), and materials from the excavations at DgRv-6 (DGRV6).

\subsection{Natural Sample Sets}

During the 2010 field season, surface cobbles were selected from a total of six 5 x 5 m grids (Analytic Units 1-6) spaced $20 \mathrm{~m}$ apart to cover the extent of the northwest portion of Parry Lagoon, which had the highest concentration of surface cobbles (Figure 2). GPS coordinates were obtained for the southwest corner of each grid so that each grid could be re-located. Within each $5 \times 5 \mathrm{~m}$ grid, all cobbles greater than $15 \mathrm{~cm}$ in diameter with flat cortical surfaces that could potentially be used as platforms for hard hammer percussion were examined (Figure 3). A sample of cobbles from the lower (glacial) deposits of the wave cut bank west of the beach surface was also collected (Glacial Units 1-3). Only cobbles on the exposed surface of the glacial till deposits of the wave cut bank were taken to minimize contributing to the erosion of the bank. The same criteria used to select the surface cobbles were also used to select materials from glacial till. Cobbles from glacial till were marked and placed in a separate large sample bag. In total 193 cobbles fit the initial collection criteria. 


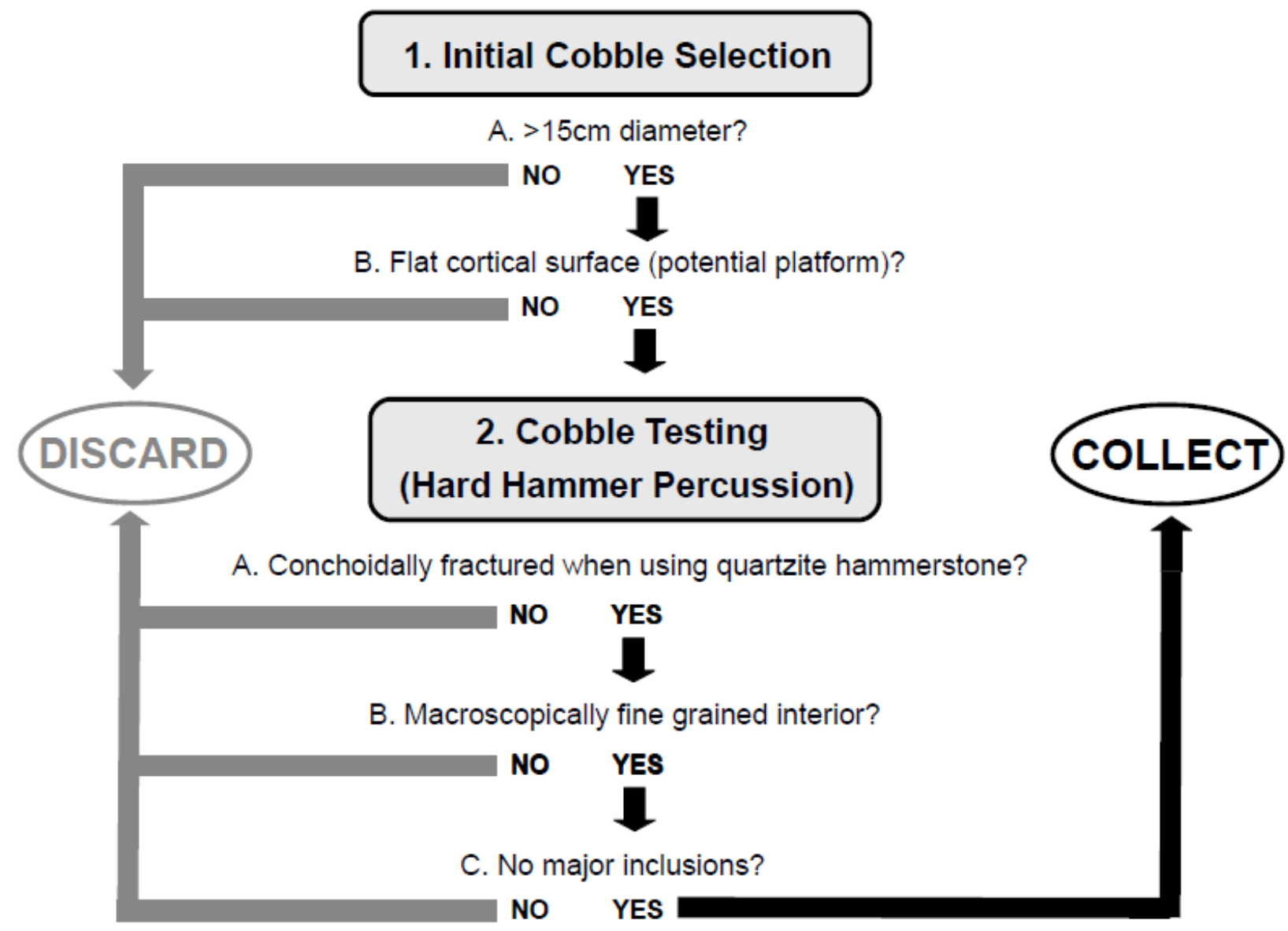

Figure 3 Cobble selection criteria flow chart.

After the initial cobble selection process, cobbles were evaluated on several criteria that have been noted as affecting toolstone quality, include fracture mechanics, grain, shape and size (e.g., Crabtree, 1972; Doelman, et al. 2008; Rorabaugh and McNabb, 2014; Whittaker, 1994; Wilson, 2007). This ensured our analyzed sample included materials that were more likely to have been selected for lithic reduction by the communities at the Dionisio Point locality. $25.3 \%(\mathrm{~N}=49)$ of all examined cobbles of sufficient size $(>15 \mathrm{~cm}$ diameter $)$ met these criteria and were analyzed.

Cobbles were tested using hard hammer percussion with a local quartzite cobblestone. Cobbles that did not flake 
when using the hammer stone were removed from the sample. As cobble cortex is a poor indicator of material quality, the exposed interior of each cobble was examined for whether or not it was macroscopically fine grained and for the absence of major inclusions that would negatively impact conchoidal fracture. All debitage from testing and tested cobbles were collected to prevent these tests from contributing to the "archaeological" record of the area. Cobbles not selected for analysis were placed in the backfill of the excavation units at DgRv-6.

\subsection{Cultural Sample Sets}

Cultural sample sets were collected from three different contexts: (1) the Coon Bay and Parry Lagoon beaches, (2) the DgRv-6 structure, and (3) the DgRv-6 midden along the Parry Lagoon shoreline. The cultural sample from the beaches consists of isolated lithic artifacts (N=7) randomly encountered and collected during the 2009 to 2011 field seasons. These artifacts had likely eroded out of site contexts from various areas of the tombolo and beaches on the Parry Lagoon and Coon Bay sides. These artifacts include reduced cores, utilized flakes, and bifaces. Debitage selected from the DgRv-6 house structure and midden for analysis was recovered during excavations at DgRv-6 in 2010 and 2012.

Debitage for analysis were selected using criteria from Sullivan and Rozen's (1985) typological approach. Lithic debris over $10 \mathrm{~g}$ in mass but where no single interior surface or point of applied force was discernible were selected for destructive analysis to minimize potential data loss for future debitage analyses. In total, 16 pieces of debitage from the house structure and midden met these criteria, and well represent the house and midden both spatially and temporally.

3.3 Methods

We employed Wave Dispersive X-Ray Fluorescence (WDXRF), despite its destructive nature, to overcome the analytical constraints in detecting light elements with nondestructive field methods, such as portable X-Ray 
Fluorescence (pXRF) (e.g. Conrey et al., 2014). Identification of crystalline volcanic rocks is based on $\mathrm{SiO}_{2}$ content, which is a compound too light to be detected reliably by pXRF. Samples were prepared for analysis by doublefusion homogenization (Johnson et al., 1999) in the Peter Hooper Geoanalytical Lab, Washington State University . Larger samples were reduced to $100-150 \mathrm{~g}$ using a hydraulic press to break the CVR cobbles into smaller pieces, samples were chipped and then ground in a tungsten carbide swing mill to prepare a homogeneous powder. Ground samples were prepared in a 2:1 ratio from $2 \mathrm{~g}$ sample and $4 \mathrm{~g}$ spec pure dilithium tetraborate (flux). The sample/flux mixture was then loaded into graphite crucibles and fused for 10 minutes at $1000^{\circ} \mathrm{C}$. Cooled samples were reground, re-fused, and polished before analysis. We determined total carbon by loss-on-ignition (Ball, 1964; Heiri et al., 2001) to corroborate instrument calculations of carbon loss used to normalize major and trace elements.

WDXRF analyses were performed on a ThermoARL Advant'XP + sequential x-ray fluorescence spectrometer for 28 elements and compounds: major elements and compounds $\mathrm{SiO}_{2}, \mathrm{TiO}_{2}, \mathrm{Al}_{2} \mathrm{O}, \mathrm{FeO}$ (total $\mathrm{Fe}$ as $\mathrm{FeO}$ ), $\mathrm{MnO}, \mathrm{MgO}$, $\mathrm{Na}_{2} \mathrm{O}, \mathrm{K}_{2} \mathrm{O}, \mathrm{CaO}$ and $\mathrm{P}_{2} \mathrm{O}_{5}$ and trace elements $\mathrm{Ni}, \mathrm{Cr}, \mathrm{Sc}, \mathrm{V}, \mathrm{Ba}, \mathrm{Rb}, \mathrm{Sr}, \mathrm{Zr}, \mathrm{Y}, \mathrm{Nb}, \mathrm{Ga}, \mathrm{Cu}, \mathrm{Zn}, \mathrm{Pb}, \mathrm{La}, \mathrm{Ce}, \mathrm{Th}$, and $\mathrm{Nd}$. Instrument performance was regularly assessed with drift monitors and 105 international certified reference materials. Replicates were analyzed every 20 samples. Limits of determination (LOD), with uncertainties expressed as 2-sigma limits, and are available from the Peter Hooper GeoAnalytical Lab at the web address (http://www.sees.wsu.edu/Geolab/note/xrfprecision.html). All elements used for this analysis fall within the limits of detection outlined by the lab.

\section{RESULTS}

All analyzed major (Table 1) and minor (Table 2) elements and compounds were present at concentrations above the analytical uncertainties determined by our instrument in each sample. Samples were identified as basalt, andesite, dacite, or rhyolite based on the silica content (Table 3) and total alkali and silica (Figure 4). Although the TAS classification includes some finer material identifications (Picrebasalt, Basanite/Tephrite, Tachybasalt, Trachyandesite, Trachyte) we emphasize the classification based on $\mathrm{SiO}_{2}$ to remain consistent with the terminology and classifications used other researchers working in the region. Samples classified as having low $\mathrm{SiO}_{2}$ content 
(basalts) have $55 \%$ or less $\mathrm{SiO}_{2}$. High $\mathrm{SiO}_{2}$ samples (andesite, dacite, and rhyolite) have a $\mathrm{SiO}_{2}$ content above $55 \%$.

\begin{tabular}{|c|c|c|c|c|c|c|c|c|c|}
\hline \multirow[t]{2}{*}{ Sample } & \multicolumn{9}{|c|}{ Major Elements (Wt. \%) } \\
\hline & $\mathrm{SiO}_{2}$ & $\mathrm{TiO}_{2}$ & $\mathrm{Al}_{2} \mathrm{O}_{4}$ & $\mathrm{FeO}^{*}$ & MnO & MgO & $\mathrm{CaO}$ & $\mathrm{Na}_{2} \mathrm{O}$ & $\mathrm{K}_{2} \mathrm{O}$ \\
\hline BEA1 & 49.42 & 1.927 & 14.34 & 12.44 & 0.240 & 7.08 & 11.30 & 2.72 & 0.36 \\
\hline BEA2 & 49.40 & 1.949 & 15.07 & 12.02 & 0.213 & 7.40 & 10.64 & 2.89 & 0.25 \\
\hline BEA3 & 69.29 & 0.434 & 16.63 & 2.30 & 0.023 & 1.19 & 2.20 & 5.02 & 2.78 \\
\hline BEA4 & 50.04 & 1.922 & 16.97 & 12.26 & 0.263 & 4.95 & 8.79 & 3.33 & 0.93 \\
\hline BEA5 & 49.68 & 2.060 & 13.19 & 14.16 & 0.190 & 7.26 & 8.97 & 4.00 & 0.32 \\
\hline BEA6 & 53.51 & 0.541 & 15.63 & 7.28 & 0.152 & 8.26 & 10.82 & 3.01 & 0.72 \\
\hline BEA7 & 58.69 & 0.907 & 18.69 & 8.14 & 0.116 & 3.32 & 5.43 & 2.39 & 2.10 \\
\hline BEA8 & 50.70 & 1.722 & 15.66 & 10.76 & 0.174 & 7.24 & 9.36 & 3.60 & 0.62 \\
\hline BEA9 & 49.89 & 1.904 & 14.25 & 12.51 & 0.228 & 6.58 & 12.31 & 2.00 & 0.16 \\
\hline BEA10 & 50.77 & 1.767 & 15.39 & 10.78 & 0.192 & 7.29 & 9.59 & 3.85 & 0.24 \\
\hline BEA11 & 70.01 & 0.725 & 14.72 & 6.22 & 0.071 & 3.19 & 0.79 & 1.46 & 2.71 \\
\hline BEA12 & 53.29 & 1.633 & 13.74 & 10.05 & 0.210 & 7.18 & 9.28 & 4.15 & 0.31 \\
\hline BEA13 & 68.33 & 0.825 & 15.05 & 6.19 & 0.063 & 2.55 & 1.51 & 2.39 & 2.99 \\
\hline BEA14 & 54.06 & 1.101 & 16.99 & 9.98 & 0.186 & 6.50 & 8.60 & 2.05 & 0.37 \\
\hline BEA15 & 50.32 & 1.465 & 14.91 & 10.83 & 0.182 & 7.80 & 11.33 & 2.91 & 0.14 \\
\hline BEA16 & 49.88 & 1.968 & 14.44 & 13.07 & 0.219 & 6.01 & 11.30 & 2.73 & 0.17 \\
\hline BEA17 & 49.87 & 1.484 & 15.97 & 10.86 & 0.196 & 7.44 & 11.23 & 2.62 & 0.18 \\
\hline BEA18 & 49.85 & 1.461 & 14.35 & 11.94 & 0.192 & 7.54 & 12.52 & 1.84 & 0.19 \\
\hline BEA19 & 45.09 & 2.358 & 15.11 & 14.73 & 0.235 & 7.03 & 14.05 & 0.97 & 0.21 \\
\hline BEA20 & 49.11 & 1.648 & 15.04 & 11.36 & 0.201 & 7.68 & 12.40 & 2.26 & 0.16 \\
\hline WCB1 & 49.85 & 1.783 & 14.20 & 12.29 & 0.201 & 7.01 & 12.55 & 1.77 & 0.20 \\
\hline WCB2 & 48.03 & 1.823 & 14.57 & 12.28 & 0.186 & 8.43 & 11.60 & 2.79 & 0.13 \\
\hline WCB3 & 50.30 & 1.707 & 14.03 & 11.49 & 0.189 & 7.21 & 12.92 & 1.90 & 0.12 \\
\hline WCB4 & 50.05 & 1.452 & 15.11 & 11.10 & 0.180 & 7.79 & 11.01 & 3.00 & 0.18 \\
\hline WCB5 & 50.02 & 1.407 & 14.31 & 11.50 & 0.188 & 7.60 & 11.47 & 2.94 & 0.45 \\
\hline WCB6 & 49.75 & 1.835 & 14.40 & 12.57 & 0.200 & 7.54 & 10.71 & 2.68 & 0.18 \\
\hline WCB7 & 49.30 & 1.544 & 15.20 & 11.04 & 0.187 & 7.57 & 12.81 & 1.99 & 0.21 \\
\hline WCB8 & 50.94 & 1.379 & 14.27 & 11.85 & 0.151 & 7.21 & 9.87 & 4.04 & 0.15 \\
\hline WCB9 & 49.23 & 1.849 & 14.15 & 12.44 & 0.207 & 7.42 & 12.12 & 2.17 & 0.25 \\
\hline WCB10 & 49.85 & 0.674 & 16.56 & 9.20 & 0.174 & 8.42 & 12.70 & 2.01 & 0.32 \\
\hline WCB11 & 48.82 & 2.351 & 15.46 & 12.28 & 0.198 & 5.97 & 12.47 & 2.12 & 0.11 \\
\hline WCB12 & 57.39 & 1.684 & 17.08 & 8.71 & 0.234 & 3.55 & 8.29 & 2.53 & 0.38 \\
\hline WCB13 & 50.29 & 2.537 & 14.10 & 12.60 & 0.191 & 6.28 & 11.04 & 2.48 & 0.25 \\
\hline WCB14 & 49.24 & 1.968 & 14.39 & 12.60 & 0.166 & 6.76 & 10.49 & 4.01 & 0.20 \\
\hline WCB15 & 49.85 & 1.866 & 14.60 & 12.40 & 0.193 & 6.36 & 12.29 & 2.16 & 0.11 \\
\hline WCB16 & 49.37 & 1.722 & 12.89 & 12.79 & 0.199 & 8.89 & 11.82 & 2.00 & 0.18 \\
\hline WCB17 & 49.84 & 1.749 & 14.76 & 12.02 & 0.213 & 7.60 & 10.74 & 2.74 & 0.19 \\
\hline WCB18 & 48.83 & 2.306 & 15.49 & 11.64 & 0.183 & 6.01 & 12.55 & 2.49 & 0.29 \\
\hline WCB19 & 52.09 & 1.215 & 18.69 & 10.78 & 0.189 & 4.76 & 7.22 & 3.42 & 1.37 \\
\hline WCB20 & 49.35 & 1.429 & 15.08 & 11.28 & 0.212 & 8.04 & 11.28 & 2.66 & 0.55 \\
\hline WCB21 & 50.03 & 1.979 & 15.73 & 12.77 & 0.198 & 7.20 & 9.06 & 2.65 & 0.21 \\
\hline WCB22 & 61.28 & 0.496 & 17.66 & 5.96 & 0.095 & 2.34 & 4.48 & 4.88 & 2.55 \\
\hline WCB23 & 52.21 & 1.813 & 13.47 & 10.74 & 0.196 & 5.17 & 13.10 & 3.12 & 0.02 \\
\hline WCB24 & 66.05 & 0.714 & 16.52 & 4.75 & 0.075 & 1.24 & 3.65 & 5.02 & 1.73 \\
\hline
\end{tabular}




\begin{tabular}{llllllllll} 
WCB25 & 50.03 & 1.548 & 15.15 & 10.82 & 0.184 & 7.27 & 12.11 & 2.51 & 0.24 \\
WCB26 & 65.80 & 0.794 & 16.65 & 6.23 & 0.145 & 2.42 & 2.77 & 2.50 & 2.46 \\
WCB27 & 49.94 & 2.086 & 16.02 & 11.24 & 0.153 & 4.82 & 11.79 & 3.71 & 0.04 \\
WCB28 & 50.40 & 1.576 & 15.43 & 11.13 & 0.233 & 7.02 & 9.90 & 3.41 & 0.78 \\
WCB29 & 50.16 & 2.488 & 13.92 & 12.94 & 0.201 & 6.29 & 11.78 & 1.90 & 0.09 \\
DBF1 & 48.66 & 1.714 & 16.18 & 11.64 & 0.178 & 7.62 & 11.82 & 1.86 & 0.16 \\
\hline
\end{tabular}

\begin{tabular}{|c|c|c|c|c|c|c|c|c|c|}
\hline \multirow[t]{2}{*}{ Sample } & \multicolumn{9}{|c|}{ Major Elements (Wt. \%) } \\
\hline & $\mathrm{SiO}_{2}$ & $\mathrm{TiO}_{2}$ & $\mathbf{A l}_{2} \mathbf{O}_{4}$ & $\mathrm{FeO}^{*}$ & MnO & MgO & $\mathrm{CaO}$ & $\mathrm{Na}_{2} \mathrm{O}$ & $\mathbf{K}_{2} \mathbf{O}$ \\
\hline DBF2 & 66.04 & 0.466 & 16.18 & 3.90 & 0.080 & 2.46 & 4.73 & 4.22 & 1.77 \\
\hline DBF3 & 48.80 & 1.928 & 15.39 & 10.99 & 0.190 & 7.34 & 12.96 & 1.99 & 0.27 \\
\hline DBF4 & 50.10 & 1.865 & 14.46 & 11.83 & 0.179 & 6.88 & 11.74 & 2.44 & 0.34 \\
\hline DBF5 & 65.85 & 0.476 & 16.30 & 3.90 & 0.080 & 2.44 & 4.80 & 4.26 & 1.74 \\
\hline DBF6 & 57.99 & 1.266 & 20.45 & 8.21 & 0.134 & 3.02 & 2.27 & 3.88 & 2.51 \\
\hline DBF7 & 60.81 & 1.079 & 23.20 & 3.39 & 0.081 & 2.48 & 4.14 & 3.18 & 1.19 \\
\hline 5DBF1 & 49.52 & 1.574 & 15.26 & 11.00 & 0.173 & 6.87 & 13.23 & 2.10 & 0.14 \\
\hline 5DBF2 & 53.72 & 1.542 & 14.15 & 9.90 & 0.141 & 4.25 & 14.89 & 1.18 & 0.09 \\
\hline 5DBF3 & 49.40 & 1.586 & 17.31 & 11.04 & 0.184 & 5.46 & 12.35 & 2.23 & 0.30 \\
\hline 5DBF4 & 49.08 & 1.851 & 14.86 & 12.07 & 0.226 & 7.10 & 11.41 & 3.01 & 0.23 \\
\hline 6DBF1 & 80.08 & 0.423 & 9.06 & 3.81 & 0.062 & 1.30 & 1.79 & 2.40 & 1.01 \\
\hline 6DBF2 & 50.56 & 0.779 & 17.56 & 10.35 & 0.186 & 7.40 & 8.30 & 3.80 & 0.92 \\
\hline 6DBF3 & 50.71 & 1.961 & 17.18 & 15.30 & 0.307 & 5.18 & 5.53 & 3.41 & 0.24 \\
\hline 6DBF4 & 49.78 & 1.211 & 15.20 & 8.60 & 0.212 & 9.54 & 11.68 & 1.20 & 2.09 \\
\hline 6DBF5 & 75.80 & 0.489 & 12.26 & 4.85 & 0.024 & 2.03 & 0.80 & 1.73 & 1.95 \\
\hline 6DBF6 & 50.37 & 1.527 & 15.17 & 10.53 & 0.177 & 6.92 & 12.97 & 2.09 & 0.11 \\
\hline DGRV6-111 & 50.16 & 1.590 & 15.05 & 11.49 & 0.186 & 7.29 & 11.43 & 2.41 & 0.24 \\
\hline DGRV6-162 & 49.94 & 1.849 & 15.37 & 12.04 & 0.131 & 6.88 & 9.85 & 2.90 & 0.85 \\
\hline DGRV6-194 & 68.28 & 0.560 & 15.88 & 5.60 & 0.078 & 2.10 & 1.85 & 3.30 & 2.16 \\
\hline DGRV6-262 & 60.71 & 0.896 & 22.88 & 6.79 & 0.091 & 2.08 & 0.49 & 1.39 & 4.45 \\
\hline DGRV6-273A & 49.31 & 1.601 & 14.93 & 11.14 & 0.186 & 7.25 & 12.93 & 2.23 & 0.22 \\
\hline DGRV6-273B & 72.06 & 0.510 & 15.00 & 3.52 & 0.038 & 1.49 & 1.38 & 3.68 & 2.10 \\
\hline DGRV6-276 & 66.49 & 0.812 & 14.23 & 8.02 & 0.100 & 3.15 & 2.37 & 3.19 & 1.08 \\
\hline DGRV6-292 & 49.90 & 1.753 & 14.13 & 12.09 & 0.202 & 7.02 & 12.79 & 1.65 & 0.30 \\
\hline DGRV6-293 & 74.45 & 0.345 & 13.14 & 2.91 & 0.096 & 0.62 & 2.66 & 4.63 & 0.97 \\
\hline DGRV6-298 & 49.21 & 2.158 & 15.28 & 11.97 & 0.179 & 5.83 & 12.53 & 2.12 & 0.39 \\
\hline DGRV6-301 & 49.29 & 1.705 & 15.24 & 12.01 & 0.196 & 7.46 & 10.99 & 2.35 & 0.57 \\
\hline DGRV6-316 & 62.80 & 0.625 & 17.07 & 4.80 & 0.098 & 2.91 & 5.31 & 4.61 & 1.49 \\
\hline DGRV6-355 & 49.40 & 2.209 & 15.19 & 11.92 & 0.184 & 5.91 & 12.38 & 2.29 & 0.30 \\
\hline DGRV6-384 & 49.91 & 1.837 & 14.17 & 12.27 & 0.200 & 7.22 & 11.95 & 1.97 & 0.29 \\
\hline DGRV6-428A & 64.93 & 0.732 & 16.69 & 6.40 & 0.384 & 2.44 & 3.23 & 1.94 & 2.91 \\
\hline DGRV6-428B & 60.99 & 0.814 & 17.68 & 4.68 & 0.085 & 2.90 & 6.99 & 4.11 & 1.45 \\
\hline DGRV6-428C & 65.67 & 0.472 & 16.37 & 3.78 & 0.079 & 2.56 & 4.84 & 4.36 & 1.69 \\
\hline
\end{tabular}

Table 1 WDXRF determinations of CVR composition for ten major elements presented as oxides ( $\mathrm{n}=83$ ). Total Fe is expressed as $\mathrm{FeO}$. LOI is carbon plus volatile loss as determined by loss-on-ignition. 


\begin{tabular}{|c|c|c|c|c|c|c|c|c|c|c|c|c|c|c|c|c|c|}
\hline \multicolumn{8}{|l|}{ Samples } & \multicolumn{10}{|c|}{ Trace Elements (ppm) } \\
\hline & $\mathrm{Ni}$ & $\mathrm{Cr}$ & Sc & $\mathbf{V}$ & $\mathbf{B a}$ & $\mathbf{R b}$ & $\mathrm{Sr}$ & $\mathrm{Zr}$ & $\mathbf{Y}$ & Nb & $\mathbf{G a}$ & $\mathbf{C u}$ & $\mathrm{Zn}$ & $\mathbf{P b}$ & La & $\mathrm{Ce}$ & Th \\
\hline BEA1 & 78 & 99 & 42 & 365 & 95 & 3 & 224 & 110 & 27 & 10.6 & 16 & 91 & 82 & 1 & 9 & 27 & 1 \\
\hline BEA2 & 102 & 153 & 43 & 387 & 68 & 4 & 221 & 113 & 30 & 10.0 & 18 & 134 & 75 & 0 & 4 & 21 & 0 \\
\hline BEA3 & 11 & 16 & 5 & 42 & 922 & 75 & 388 & 124 & 5 & 3.1 & 21 & 9 & 55 & 15 & 18 & 27 & 7 \\
\hline BEA4 & 24 & 10 & 38 & 339 & 337 & 27 & 398 & 224 & 58 & 9.1 & 21 & 122 & 121 & 9 & 19 & 50 & 2 \\
\hline BEA5 & 70 & 73 & 43 & 387 & 81 & 8 & 383 & 114 & 29 & 9.9 & 15 & 71 & 61 & 0 & 8 & 22 & 1 \\
\hline BEA6 & 60 & 285 & 42 & 234 & 568 & 17 & 149 & 24 & 15 & 0.7 & 13 & 4 & 77 & 2 & 2 & 8 & 0 \\
\hline BEA7 & 11 & 17 & 25 & 171 & 694 & 49 & 644 & 106 & 24 & 4.1 & 18 & 48 & 94 & 5 & 7 & 24 & 3 \\
\hline BEA8 & 85 & 253 & 37 & 319 & 178 & 9 & 243 & 100 & 25 & 9.1 & 17 & 378 & 84 & 2 & 8 & 19 & 0 \\
\hline BEA9 & 76 & 95 & 40 & 359 & 86 & 3 & 178 & 107 & 26 & 9.4 & 19 & 159 & 101 & 1 & 7 & 24 & 2 \\
\hline BEA10 & 91 & 272 & 40 & 326 & 57 & 5 & 153 & 106 & 21 & 9.1 & 17 & 24 & 94 & 2 & 8 & 13 & 0 \\
\hline BEA11 & 17 & 42 & 20 & 160 & 1357 & 56 & 108 & 113 & 20 & 6.0 & 16 & 62 & 73 & 8 & 15 & 30 & 4 \\
\hline BEA12 & 90 & 198 & 42 & 341 & 91 & 9 & 379 & 92 & 25 & 8.1 & 14 & 102 & 62 & 0 & 8 & 18 & 1 \\
\hline BEA13 & 32 & 41 & 20 & 178 & 1150 & 59 & 284 & 95 & 16 & 5.0 & 16 & 65 & 96 & 16 & 10 & 21 & 4 \\
\hline BEA14 & 39 & 127 & 33 & 280 & 192 & 8 & 426 & 67 & 17 & 3.4 & 18 & 78 & 109 & 5 & 8 & 17 & 2 \\
\hline BEA15 & 100 & 316 & 41 & 323 & 35 & 2 & 207 & 83 & 23 & 7.6 & 15 & 131 & 87 & 2 & 9 & 19 & 2 \\
\hline BEA16 & 62 & 81 & 44 & 404 & 66 & 2 & 375 & 115 & 32 & 8.7 & 21 & 87 & 104 & 0 & 8 & 19 & 1 \\
\hline BEA17 & 93 & 257 & 36 & 318 & 64 & 3 & 230 & 86 & 23 & 7.5 & 17 & 38 & 81 & 2 & 7 & 17 & 1 \\
\hline BEA18 & 95 & 170 & 44 & 322 & 50 & 4 & 164 & 80 & 22 & 7.0 & 16 & 29 & 87 & 1 & 7 & 13 & 0 \\
\hline BEA19 & 108 & 122 & 51 & 447 & 31 & 1 & 91 & 138 & 33 & 12.0 & 25 & 51 & 120 & 1 & 18 & 33 & 1 \\
\hline BEA20 & 100 & 260 & 42 & 341 & 46 & 4 & 180 & 93 & 25 & 9.2 & 19 & 179 & 87 & 2 & 9 & 20 & 0 \\
\hline WCB1 & 86 & 130 & 42 & 358 & 98 & 2 & 188 & 102 & 26 & 8.9 & 17 & 122 & 94 & 1 & 9 & 19 & 1 \\
\hline WCB2 & 92 & 253 & 43 & 356 & 30 & 1 & 115 & 108 & 27 & 9.9 & 19 & 84 & 76 & 0 & 10 & 21 & 1 \\
\hline WCB3 & 95 & 262 & 44 & 354 & 33 & 2 & 163 & 97 & 26 & 9.3 & 17 & 164 & 79 & 2 & 9 & 17 & 0 \\
\hline WCB4 & 99 & 309 & 42 & 317 & 38 & 2 & 229 & 84 & 23 & 8.1 & 16 & 144 & 84 & 0 & 6 & 18 & 0 \\
\hline WCB5 & 97 & 192 & 44 & 327 & 76 & 9 & 322 & 75 & 21 & 6.0 & 16 & 56 & 91 & 2 & 6 & 15 & 1 \\
\hline WCB6 & 85 & 127 & 42 & 361 & 109 & 5 & 226 & 101 & 26 & 9.8 & 16 & 117 & 98 & 0 & 8 & 17 & 1 \\
\hline WCB7 & 105 & 308 & 41 & 329 & 44 & 5 & 176 & 87 & 24 & 7.7 & 16 & 156 & 81 & 1 & 8 & 17 & 0 \\
\hline WCB8 & 81 & 223 & 41 & 288 & 60 & 4 & 245 & 77 & 21 & 6.6 & 15 & 3 & 73 & 3 & 4 & 6 & 1 \\
\hline WCB9 & 92 & 137 & 42 & 369 & 65 & 2 & 193 & 107 & 27 & 9.6 & 20 & 170 & 100 & 2 & 8 & 21 & 0 \\
\hline WCB10 & & 243 & 48 & 285 & 100 & 6 & 274 & 34 & 16 & 1.3 & 16 & 125 & 74 & 1 & 5 & 8 & 1 \\
\hline WCB11 & & 140 & 38 & 395 & 43 & 1 & 241 & 144 & 31 & 12.7 & 22 & 228 & 108 & 3 & 10 & 29 & 1 \\
\hline WCB12 & & 181 & 44 & 324 & 142 & 10 & 245 & 94 & 26 & 8.5 & 17 & 52 & 39 & 1 & 4 & 16 & 1 \\
\hline WCB13 & 82 & 151 & 40 & 409 & 95 & 6 & 286 & 150 & 31 & 14.6 & 23 & 230 & 112 & 1 & 12 & 31 & 1 \\
\hline
\end{tabular}




\begin{tabular}{|c|c|c|c|c|c|c|c|c|c|c|c|c|c|c|c|c|}
\hline \multirow{2}{*}{$\stackrel{\text { Samples }}{\mathrm{Ni}}$} & \multicolumn{16}{|c|}{ Trace Elements (ppm) } \\
\hline & $\mathrm{Cr}$ & Sc & $\mathbf{V}$ & Ba & $\mathbf{R b}$ & $\mathrm{Sr}$ & $\mathrm{Zr}$ & $\mathbf{Y}$ & Nb & $\mathbf{G a}$ & $\mathbf{C u}$ & $\mathbf{Z n}$ & $\mathbf{P b}$ & $\mathbf{L a}$ & $\mathrm{Ce}$ & Th \\
\hline WCB14 80 & 144 & 44 & 383 & 51 & 2 & 127 & 112 & 30 & 11.0 & 19 & 25 & 55 & 0 & 8 & 23 & 1 \\
\hline WCB15 79 & 105 & 40 & 374 & 35 & 2 & 188 & 107 & 28 & 9.0 & 18 & 182 & 95 & 1 & 8 & 24 & 1 \\
\hline WCB16 133 & 181 & 45 & 362 & 64 & 3 & 170 & 93 & 25 & 8.2 & 18 & 64 & 94 & 0 & 4 & 19 & 0 \\
\hline WCB17 81 & 95 & 41 & 355 & 54 & 3 & 207 & 99 & 26 & 8.9 & 20 & 130 & 98 & 3 & 7 & 23 & 0 \\
\hline WCB18 $_{71}$ & 144 & 38 & 392 & 61 & 4 & 217 & 137 & 31 & 12.8 & 21 & 552 & 63 & 0 & 11 & 27 & 1 \\
\hline${ }^{\text {WCB19 }}{ }_{12}$ & 9 & 31 & 297 & 273 & 28 & 338 & 65 & 29 & 3.1 & 19 & 82 & 89 & 1 & 10 & 23 & 1 \\
\hline WCB20 100 & 176 & 43 & 323 & 132 & 11 & 268 & 75 & 21 & 6.5 & 15 & 34 & 82 & 0 & 3 & 17 & 1 \\
\hline WCB21 101 & 179 & 43 & 381 & 51 & 5 & 265 & 111 & 31 & 9.6 & 17 & 187 & 101 & 2 & 8 & 20 & 2 \\
\hline WCB22 6 & 3 & 11 & 96 & 875 & 36 & 601 & 91 & 17 & 4.7 & 17 & 2 & 64 & 4 & 13 & 27 & 2 \\
\hline WCB23 68 & 90 & 37 & 366 & 18 & 1 & 323 & 103 & 26 & 8.5 & 21 & 436 & 73 & 3 & 8 & 19 & 1 \\
\hline WCB24 $_{2}$ & 3 & 15 & 42 & 944 & 38 & 308 & 212 & 42 & 7.0 & 18 & 2 & 38 & 5 & 15 & 43 & 2 \\
\hline WCB25 104 & 306 & 42 & 331 & 51 & 6 & 179 & 86 & 25 & 8.0 & 17 & 169 & 72 & 0 & 6 & 19 & 1 \\
\hline WCB26 15 & 32 & 23 & 154 & 1213 & 46 & 529 & 93 & 28 & 4.7 & 16 & 48 & 81 & 3 & 15 & 28 & 2 \\
\hline WCB27 70 & 166 & 35 & 366 & 28 & 2 & 93 & 124 & 29 & 12.0 & 22 & 121 & 74 & 0 & 10 & 29 & 1 \\
\hline WCB28 107 & 310 & 42 & 327 & 101 & 39 & 356 & 87 & 25 & 8.1 & 18 & 17 & 72 & 1 & 4 & 15 & 0 \\
\hline WCB29 82 & 140 & 39 & 406 & 47 & 2 & 219 & 146 & 31 & 14.3 & 21 & 230 & 109 & 1 & 9 & 30 & 1 \\
\hline DBF1 100 & 269 & 42 & 338 & 35 & 2 & 158 & 104 & 26 & 9.7 & 20 & 100 & 87 & 1 & 8 & 25 & 1 \\
\hline DBF2 46 & 46 & 10 & 76 & 681 & 28 & 670 & 122 & 15 & 3.7 & 18 & 26 & 57 & 8 & 14 & 27 & 2 \\
\hline DBF3 110 & 245 & 42 & 370 & 80 & 5 & 249 & 110 & 29 & 9.8 & 20 & 20 & 79 & 1 & 7 & 23 & 1 \\
\hline DBF4 111 & 253 & 40 & 364 & 87 & 6 & 204 & 106 & 26 & 9.2 & 18 & 19 & 93 & 1 & 8 & 17 & 0 \\
\hline DBF5 42 & 43 & 9 & 80 & 666 & 27 & 682 & 123 & 14 & 4.7 & 18 & 26 & 57 & 8 & 15 & 29 & 3 \\
\hline DBF6 9 & 21 & 29 & 166 & 1221 & 47 & 405 & 157 & 29 & 5.8 & 20 & 34 & 109 & 6 & 14 & 35 & 2 \\
\hline DBF7 2 & 45 & 32 & 286 & 449 & 25 & 317 & 108 & 27 & 3.5 & 20 & 13 & 71 & 7 & 4 & 13 & 2 \\
\hline 5DBF1 105 & 312 & 42 & 332 & 41 & 3 & 179 & 90 & 25 & 7.7 & 18 & 166 & 78 & 0 & 7 & 21 & 1 \\
\hline 5DBF2 81 & 248 & 35 & 306 & 54 & 3 & 88 & 91 & 23 & 8.7 & 22 & 156 & 52 & 1 & 9 & 22 & 0 \\
\hline 5DBF3 63 & 82 & 35 & 326 & 90 & 6 & 250 & 88 & 23 & 7.2 & 20 & 136 & 82 & 1 & 8 & 19 & 1 \\
\hline 5DBF4 87 & 180 & 42 & 344 & 78 & 2 & 187 & 106 & 28 & 10.1 & 19 & 73 & 113 & 0 & 10 & 24 & 3 \\
\hline 6DBF1 7 & 20 & 13 & 90 & 314 & 21 & 129 & 66 & 16 & 3.2 & 11 & 43 & 64 & 4 & 8 & 13 & 2 \\
\hline 6DBF2 40 & 120 & 40 & 285 & 810 & 11 & 467 & 27 & 16 & 1.6 & 14 & 81 & 75 & 1 & 4 & 9 & 0 \\
\hline 6DBF3 98 & 180 & 39 & 351 & 169 & 7 & 228 & 110 & 26 & 9.7 & 21 & 138 & 100 & 0 & 8 & 22 & 1 \\
\hline 6DBF4 108 & 356 & 36 & 318 & 3413 & 98 & 684 & 65 & 20 & 11.2 & 17 & 199 & 84 & 2 & 21 & 45 & 3 \\
\hline 6DBF5 22 & 35 & 14 & 115 & 576 & 40 & 222 & 80 & 14 & 4.3 & 14 & 38 & 87 & 7 & 13 & 21 & 2 \\
\hline 6DBF6 104 & 306 & 42 & 324 & 40 & 2 & 168 & 87 & 24 & 9.0 & 18 & 158 & 79 & 0 & 8 & 16 & 1 \\
\hline DGRV6-111 94 & 170 & 41 & 331 & 59 & 2 & 276 & 92 & 25 & 8.5 & 17 & 43 & 89 & 1 & 6 & 16 & 1 \\
\hline DGRV6-162 79 & 149 & 39 & 335 & 190 & 20 & 394 & 104 & 25 & 9.1 & 16 & 2 & 63 & 1 & 4 & 15 & 1 \\
\hline DGRV6-194 30 & 53 & 10 & 83 & 747 & 72 & 319 & 146 & 20 & 10.0 & 17 & 18 & 84 & 12 & 20 & 46 & 8 \\
\hline
\end{tabular}




\begin{tabular}{|c|c|c|c|c|c|c|c|c|c|c|c|c|c|c|c|c|}
\hline \multirow{2}{*}{$\begin{array}{l}\text { Samples } \\
\text { Ni }\end{array}$} & \multicolumn{16}{|c|}{ Trace Elements (ppm) } \\
\hline & $\mathrm{Cr}$ & Sc & $\mathbf{V}$ & $\mathbf{B a}$ & $\mathbf{R b}$ & $\mathbf{S r}$ & $\mathrm{Zr}$ & $\mathbf{Y}$ & Nb & $\mathbf{G a}$ & $\mathbf{C u}$ & $\mathbf{Z n}$ & $\mathbf{P b}$ & $\mathbf{L a}$ & $\mathbf{C e}$ & Th \\
\hline DGRV6-262 27 & 64 & 18 & 178 & 2002 & 135 & 115 & 144 & 20 & 10.8 & 26 & 31 & 83 & 16 & 30 & 57 & 11 \\
\hline DGRV6-273A 102 & 283 & 43 & 338 & 59 & 4 & 199 & 92 & 26 & 8.9 & 17 & 132 & 88 & 2 & 9 & 18 & 2 \\
\hline DGRV6-273B 17 & 45 & 8 & 56 & 585 & 63 & 252 & 189 & 20 & 10.2 & 16 & 8 & 58 & 11 & 22 & 41 & 7 \\
\hline DGRV6-276 8 & 9 & 16 & 108 & 570 & 43 & 392 & 101 & 33 & 4.9 & 15 & 10 & 106 & 5 & 13 & 25 & 3 \\
\hline DGRV6-292 86 & 132 & 41 & 353 & 79 & 5 & 176 & 101 & 27 & 9.0 & 18 & 111 & 92 & 2 & 4 & 18 & 1 \\
\hline DGRV6-293 3 & 4 & 15 & 26 & 535 & 18 & 224 & 116 & 36 & 3.6 & 12 & 64 & 153 & 58 & 10 & 25 & 3 \\
\hline DGRV6-298 72 & 176 & 38 & 366 & 166 & 10 & 240 & 131 & 30 & 13.4 & 21 & 153 & 105 & 0 & 8 & 28 & 2 \\
\hline DGRV6-30194 & 222 & 41 & 343 & 240 & 11 & 272 & 99 & 24 & 9.1 & 17 & 438 & 93 & 1 & 7 & 13 & 0 \\
\hline DGRV6-316 44 & 55 & 10 & 92 & 611 & 19 & 788 & 153 & 18 & 6.3 & 19 & 26 & 73 & 7 & 18 & 39 & 3 \\
\hline DGRV6-355 73 & 176 & 38 & 373 & 93 & 6 & 269 & 131 & 29 & 11.7 & 20 & 119 & 91 & 1 & 15 & 29 & 1 \\
\hline DGRV6-384 87 & 132 & 44 & 370 & 186 & 6 & 189 & 100 & 26 & 9.4 & 19 & 52 & 94 & 1 & 8 & 21 & 1 \\
\hline DGRV6-428A $_{13}$ & 22 & 24 & 165 & 2256 & 64 & 321 & 113 & 34 & 4.1 & 17 & 44 & 78 & 7 & 18 & 39 & 3 \\
\hline DGRV6-428B 25 & 20 & 11 & 114 & 445 & 15 & 1944 & 135 & 12 & 3.6 & 22 & 40 & 63 & 5 & 24 & 59 & 3 \\
\hline DGRV6-428C $_{44}$ & 46 & 9 & 79 & 610 & 25 & 719 & 115 & 14 & 3.9 & 17 & 25 & 55 & 8 & 16 & 30 & 1 \\
\hline
\end{tabular}

Table 2 WDXRF determinations of CVR composition for 18 trace elements $(n=83)$ 


\begin{tabular}{|c|c|c|c|}
\hline Natural Samples & $(n=49)$ & Cultural Samples & $(n=34)$ \\
\hline Basalt & 41 & Basalt & 19 \\
\hline Andesite & 3 & Andesite & 5 \\
\hline Dacite & 3 & Dacite & 6 \\
\hline Rhyolite & 2 & Rhyolite & 4 \\
\hline Low $\mathrm{SiO}_{2}$ & 41 & Low $\mathrm{SiO}_{2}$ & 19 \\
\hline High $\mathrm{SiO}_{2}$ & 8 & High $\mathrm{SiO}_{2}$ & 15 \\
\hline Percent (Low/High) & $84 / 16$ & Percent (Low/High) & $56 / 44$ \\
\hline
\end{tabular}

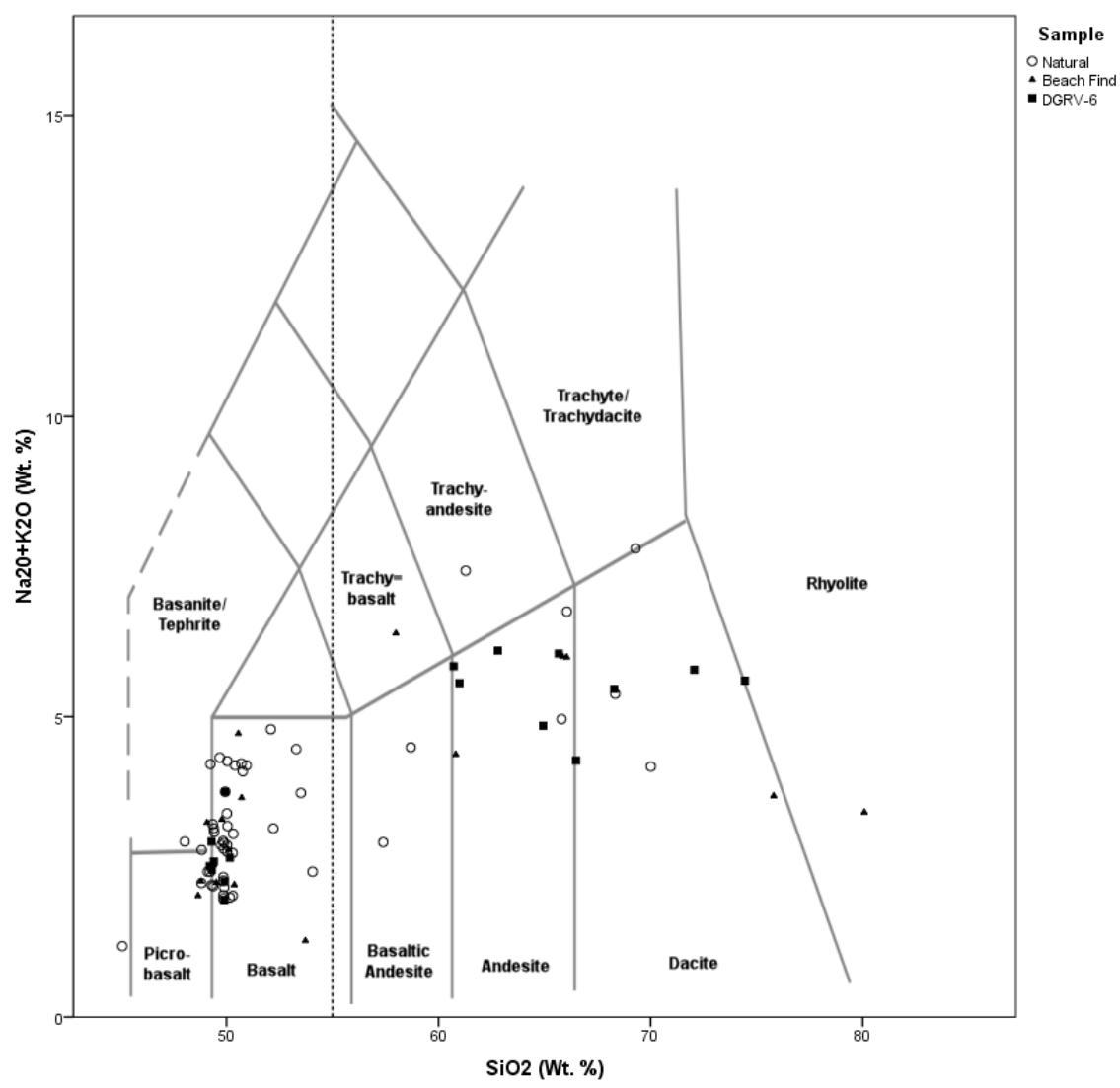

Table 3 Material types comprising natural control and cultural sample groups. Based on silica content.

Figure 4 Total Alkali Silica (TAS) Classification LeMaitre Plot

Bivariate plots were generated comparing the major and trace elements for all samples in order to show groupings of 
high $\mathrm{SiO}_{2}$ materials and low $\mathrm{SiO}_{2}$ materials across sample sets and to identify elements to be used in statistical analyses. The major elements, excluding $\mathrm{SiO}_{2}$, that were determined to be the most effective for discriminating between the two material type groups include: $\mathrm{TiO}_{2}, \mathrm{MnO}, \mathrm{MgO}, \mathrm{Na}_{2} \mathrm{O}, \mathrm{CaO}$, and $\mathrm{K}_{2} \mathrm{O}$. Trace elements with the clearest distinctions are $\mathrm{Ni}, \mathrm{Cr}, \mathrm{Sc}$, and $\mathrm{V}$.

The natural control samples $(n=49)$ were mostly basalt $(n=41)$, but also included a small number of andesite $(n=3)$, dacite $(n=3)$, and rhyolite $(n=2)$ samples (Table 3). The low $\mathrm{SiO}_{2}$ samples account for $84 \%$ of the samples in the natural control sample group, while high $\mathrm{SiO}_{2}$ samples comprised the remaining $16 \%$. The cultural samples $(\mathrm{n}=34)$, which include the beach finds (DBF) and DGRV6 sample groups, also contain basalt $(n=19)$, andesite $(n=5)$, dacite $(\mathrm{n}=6)$, and rhyolite $(\mathrm{n}=4)$. However, the ratio of low $\mathrm{SiO}_{2}$ samples to high $\mathrm{SiO}_{2}$ samples is much different, with low $\mathrm{SiO}_{2}$ samples accounting for $56 \%$ of the cultural samples and high $\mathrm{SiO}_{2}$ samples making up the other $44 \%$.

Differences in the frequency of high and low $\mathrm{SiO}_{2} \mathrm{CVR}$ in the natural and control samples are highly significant at a 0.01 level based on a Fisher's Exact test $(\mathrm{p}=0.0012)$.

Elements other than $\mathrm{SiO}_{2}$ were also found to be useful to distinguish between CVR classes. $\mathrm{MgO}$ was found to be the best element for discriminating CVR classes. The bivariate plots in Figure 5 show the distinction between samples in the low $\mathrm{SiO}_{2}$ material group and the high $\mathrm{SiO}_{2}$ using the elements $\mathrm{MnO}$ and $\mathrm{MgO}$. These plots also reveal that there are samples from both the natural control and cultural sample sets in both of the material groups.

$\mathrm{As}_{\mathrm{SiO}_{2}}$ content increases $\mathrm{MgO}$ content decreases as a result of magmatic differentiation (Jaupert and Tait 2012). All low $\mathrm{SiO}_{2}$ samples have an $\mathrm{MgO}$ content above 4 percent, while the high $\mathrm{SiO}_{2}$ samples have $\mathrm{MgO}$ content below 4 percent. This element can be reliably detected on pXRF machines and would be a good field proxy for silica content for these materials but not all CVR. As there is a complex relationship between $\mathrm{SiO}_{2}$ and $\mathrm{MgO}$ content due to magmatic differentiation processes, using $\mathrm{pXRF}$ one would only be able to distinguish between low and high silica material groups, not specific $\mathrm{SiO}_{2}$ content. The trace elements that were identified to be the most important for discriminating the two material type groups include: Ni, Cr, Sc, and V. Figure 6 shows that the distinction between 
the high quality and low quality tool stone is also evident in these trace elements. 


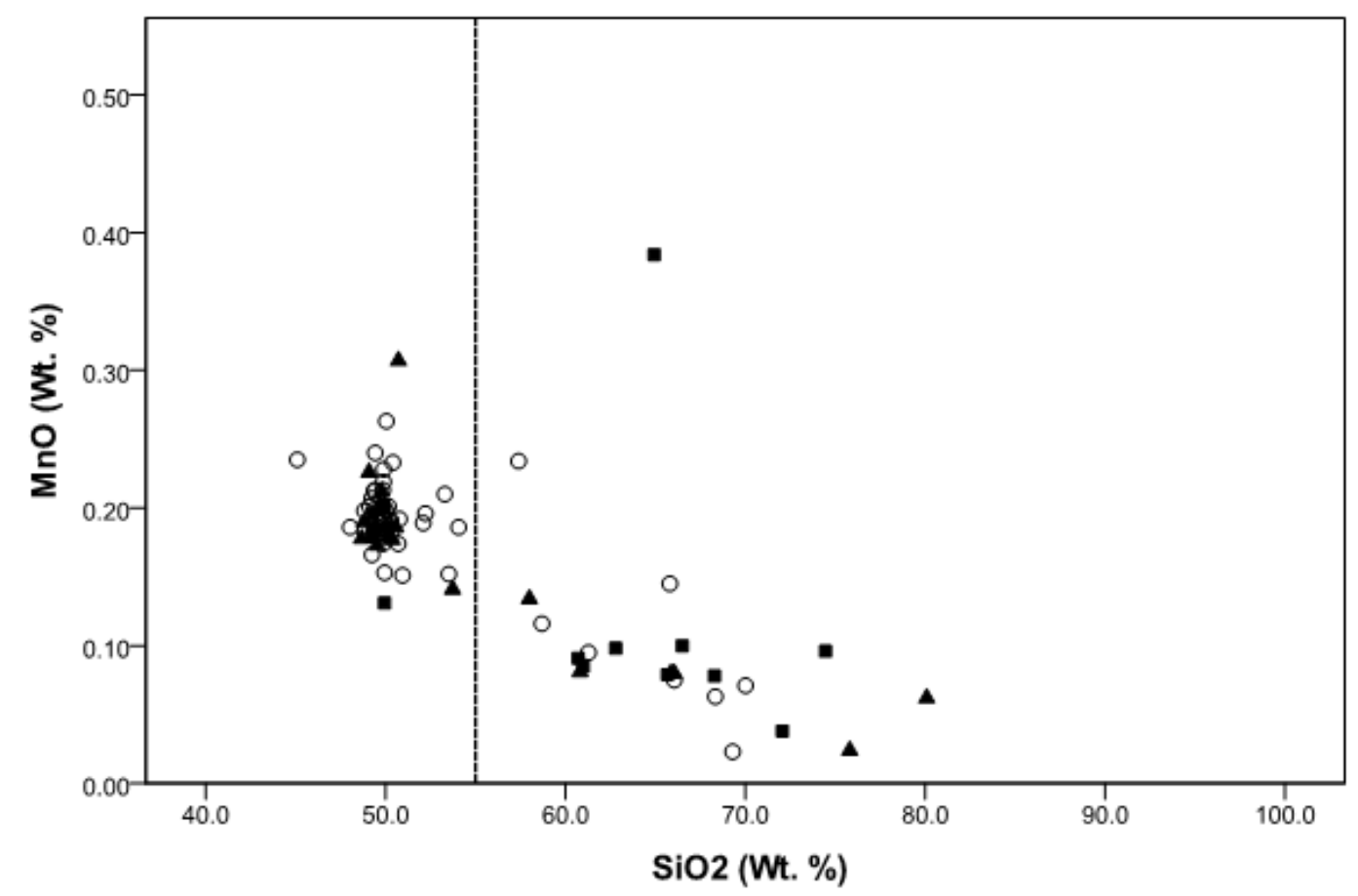

Sample

O Natural

$\Delta$ Beach Find

- DgRv-6

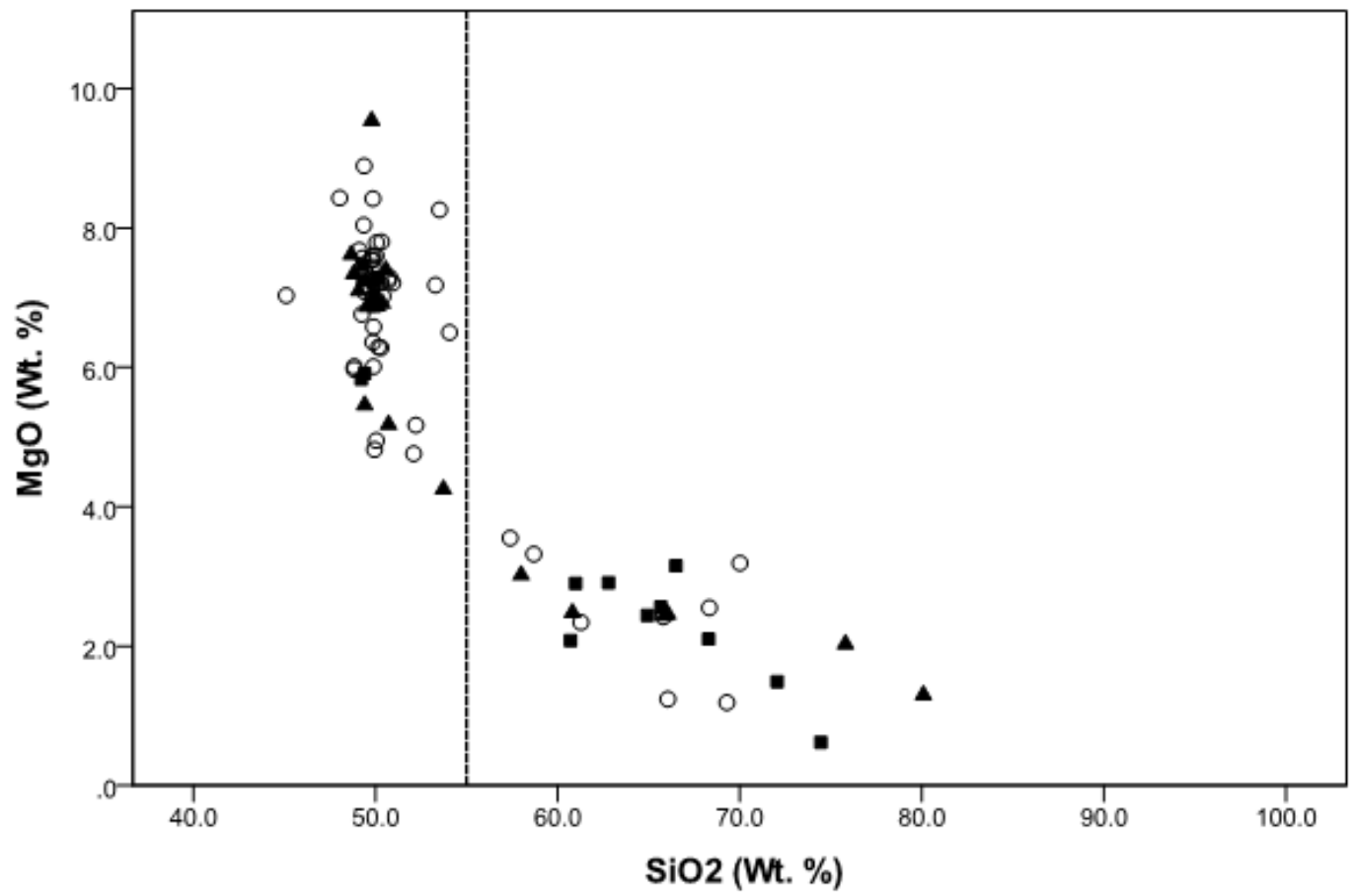

Sample

O Natural

A Beach Find

- DgRv-6

Figure 5 Bivariate plots comparing $\mathrm{SiO}_{2}$ vs $\mathrm{MnO}$ (top) and $\mathrm{SiO}_{2}$ vs $\mathrm{MgO}$ (bottom) for all sample groups. 

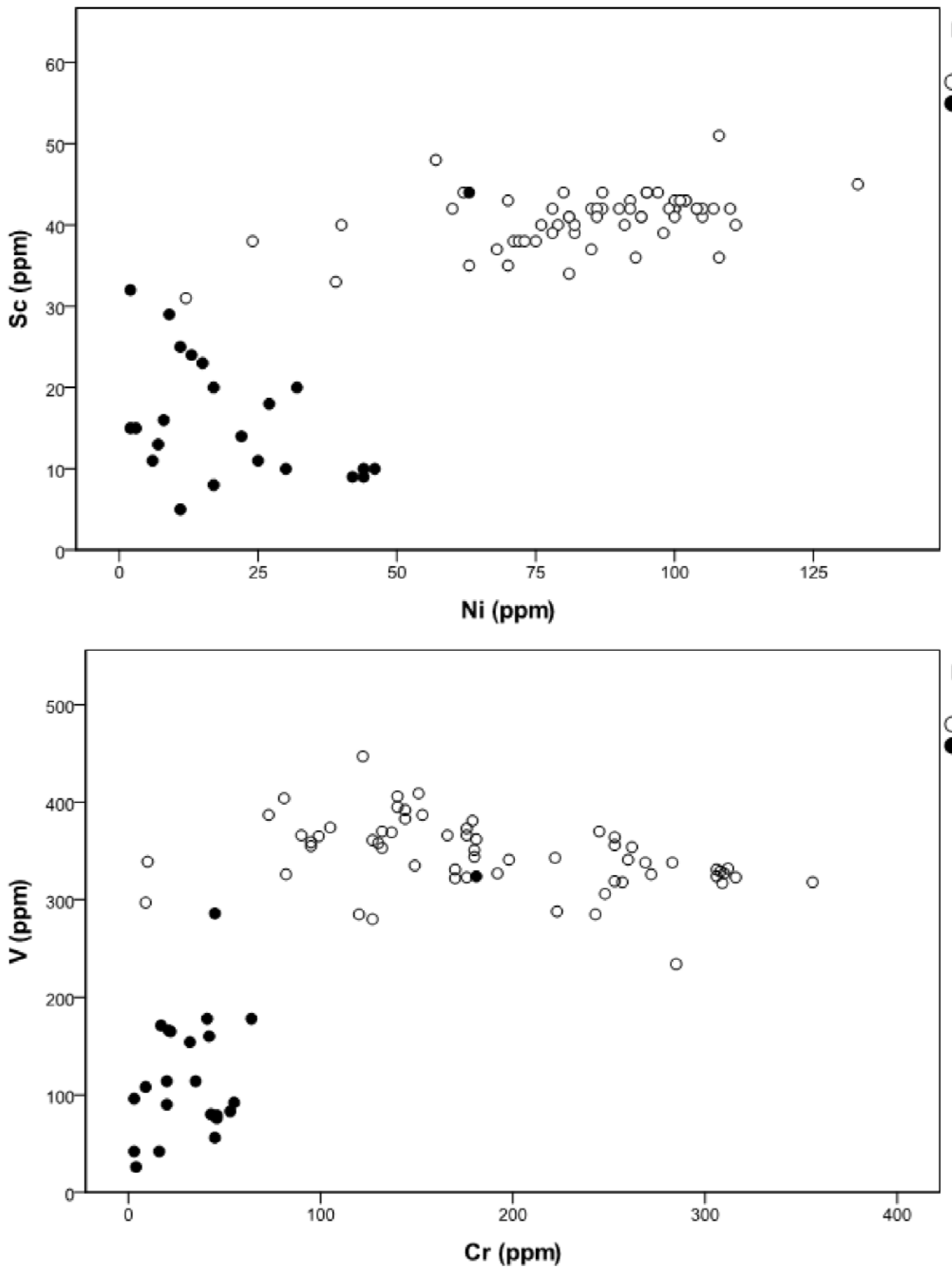

Figure 6 Bivariate plots comparing Ni vs Sc (top) and Cr vs V (bottom) for all sample groups.

To verify that the samples were forming two distinct material quality groups a Linear Discriminant Analysis (LDA) was performed on all samples using the major and trace elements, excluding $\mathrm{SiO}_{2}$ (Table 4). The results of our LDA were highly significant at a 0.01 level (Wilks' Lambda $=0.073, \chi 2=200.39, \mathrm{df}=9, \mathrm{p}=0.001$ ). This demonstrates that 
the samples from the Dionisio Point locality form two distinct groups, a low percentage $\mathrm{SiO}_{2}$ material type (Material Group 1) and a high percentage $\mathrm{SiO}_{2}$ material type (Material Group 2) with membership predicted by their constituent major and trace elements. Using major elements $100 \%$ of the samples were correctly classified into the high and low $\mathrm{SiO}_{2}$ content groups. The analysis using trace elements correctly classified samples into the high and low $\mathrm{SiO}_{2}$ content groups with one exception.

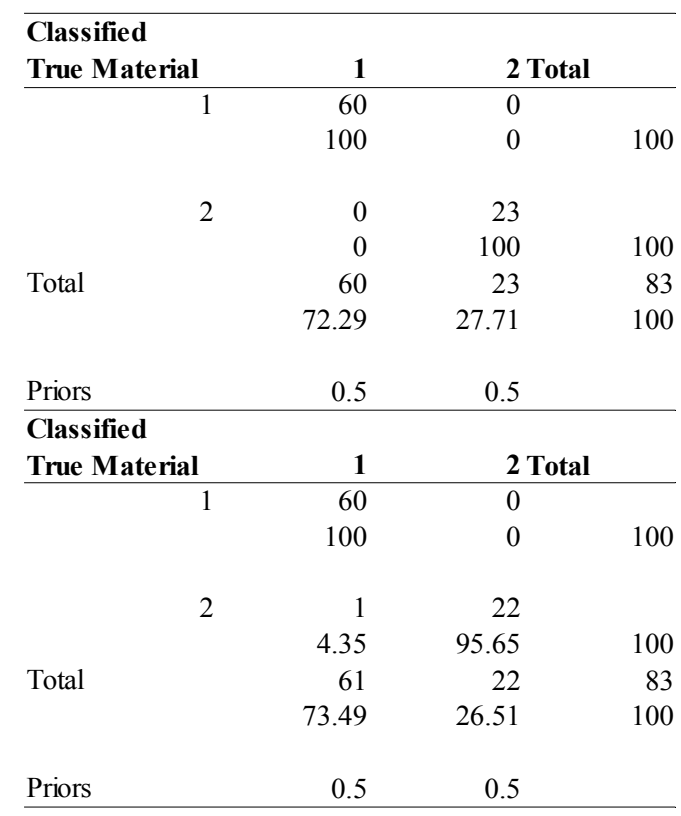

Table 4 Linear discriminant analysis of low (Material group 1) and high (Material group 2) $\mathrm{SiO}_{2}$ content materials using all major (top) and trace (bottom) elements.

\section{CONCLUSIONS}

With this study we set out to answer two core questions. First, was high quality toolstone available at the Dionisio Point locality? By directly measuring $\mathrm{SiO}_{2}$ content, we were able to assess the quality of toolstone readily available at the Dionisio Point locality. Our analyses show that basalt was the dominant material type for cobbles locally available at Parry's Lagoon. However, higher quality (higher $\mathrm{SiO}_{2}$ content) CVR toolstone including andesite, dacite, and rhylote were also present in the natural samples. Although low quality toolstone was prevalent, high quality 
toolstone was clearly accessible and locally utilized at Dionisio Point.

Our second question concerned whether there is evidence of selection for higher quality materials. The presence of basalt, andesite, dacite, and rhyolite samples in the cultural data sets reveals that precontact communities in the Dionisio Point locality were utilizing the full range of locally available lithic source materials, but with a preference for high $\mathrm{SiO}_{2}$ content materials. As the range of toolstone locally available at Parry's Lagoon includes basalt, dacite, andesite, and rhyloite, it is entirely possible that primarily local cobbles were utilized as toolstone at DgRv-6. In contrast to previous interpretations of toolstone acquisition (eg., Close, 2006), for Dionisio Point there is no evidence to support that the majority of raw toolstone was acquired through direct lithic procurement at distant locations such as Watts Point, British Columbia. However, very high quality toolstone as indicated by unusually high $\mathrm{SiO}_{2}$ content was present in the debitage from the artifact assemblages (specifically samples DGRV6-273B and DGRV6-293) but absent from the natural controls. This may indicate some use of extralocal lithic sources, whether the result of down the line trade or directed lithic procurement (Rorabaugh and McNabb, 2014). It is clear that Coast Salish peoples engaged in directed lithic procurement of exotic toolstone for reasons including ideology (e.g. Reimer, 2012), but complex trade relations, kin networks, and pilgrimages do not have to be invoked to account for the toolstone procured for many daily practices.

Although WDXRF is destructive, which may limit its applicability to many archaeological assemblages, our findings suggest that other elements can act as proxies for silica content. $\mathrm{MgO}, \mathrm{MnO}$, and trace elements can be used with pXRF and EDXRF to distinguish between high and low silica material groups in the absence of actual $\mathrm{SiO}_{2}$ data. Although WDXRF provides more accurate and precise results, we suggest that these elements can be used as a proxy for $\mathrm{SiO}_{2}$ content. This means that non-destructive analyses to examine relative toolstone material quality are feasible in the Salish Sea and other regions where CVR toolstones are prevalent. This is important for contexts where destructive methods may not be permitted due to heritage purposes but researchers have considerable questions relating to lithic technological organization. However, we argue that researchers should still use WDXRF if their research questions require the identification of specific CVR material types. 


\section{ACKNOWLEDGEMENTS}

The authors thank Richard Rupp at the Washington State University Geoanalytical Lab (WSUGAL) and Craig Skinner at the Northwest Obsidian Research Laboratory (NWORL). Funding for elemental analyses was provided by grants to Adam N. Rorabaugh by the Association for Washington Archaeology and Don F. Crabtree lithics scholarship committee. Fieldwork at the Dionisio Point locality has been made possible through the generous support of the Penelakut First Nation and BC Parks. Funding for fieldwork was provided by grants to Grier from the National Science Foundation (Grant No. 1062615) and Wenner-Gren Foundation for Anthropological Research (Grant No. 7961). 


\section{REFERENCES CITED}

Ames, C. (2009). From Chipped to Ground: the spatio-temporal systematics of 9000 years of archaeological change in southwest British Columbia. Unpublished Master's Thesis, McGill University. Montreal, Ontario.

Ames, K. (1994). The Northwest Coast: Complex Hunter-Gatherers, Ecology, and Social Evolution. Annual Review of Anthropology, 23, 209-229

Ames, K. (1995). Chiefly Power and Household Production on the Northwest Coast. In Price, T. D. and Feinman, G. M., (Eds.) Foundations of Social Inequality (pp. 155-187.) New York: Plenum Press.

Ames, K. (2010). On the Evolution of the Human Capacity for Inequality and/or Egalitarianism. In Price, T. D. and Feinman, G. M. (Eds.) Pathways to Power. (pp. 15-44.) New York: Springer.

Ames, K. and Maschner, H. D. G. (1999). Peoples of the Northwest Coast: Their Archaeology and Prehistory. London: Thames and Hudson.

Angelbeck, B., and Grier, C. (2012). Anarchism and the Archaeology of Anarchic Societies:

Resistance to Centralization in the Coast Salish Region of the Pacific Northwest Coast.

Current Anthropology Current Anthropology, 53(5), 547-587.

Ball, D. F. (1964). Loss-on-ignition as an Estimation of Organic Matter and Organic Carbon in Non-Calcareous Soils. Journal of Soil Science, 15, 84-92.

Bakewell, E. F. (1996). Petrographic and Geochemical Source-Modeling of Volcanic Lithics from Archaeological Contexts: A Case Study from British Camp, San Juan Island, Washington. Geoarchaeology, 11(2),119-140. 
Bakewell, E. F. (2005). The Archaeopetrology of Vitrophyric Toolstones, With Applications to Archaeology in the Pacific Northwest. Unpublished Doctoral Dissertation, University of Washington, Seattle, Washington.

Beattie, O. B. (1981). An Analysis of Prehistoric Human Skeletal Material from the Gulf of Georgia Region of British Columbia. Unpublished Doctoral Dissertation, Simon Fraser University. Burnaby, British Columbia.

Bilton, D. H. (2014). Northern, Central, Diversified, Specialized: The Archaeology of Fishing Adaptations in the Gulf of Georgia (Salish Sea), British Columbia. Unpublished Doctoral Dissertation. University of Toronto. Toronto, Ontario.

Burley, D. V. (1980). Marpole: Anthropological Reconstructions of a Prehistoric Northwest Coast Culture Type. Department of Archaeology Simon Fraser University Publication No. 8.

Burley, D. V. and Knusel, C. (1989). Burial Patterns Archaeological Interpretation: Problems in the Recognition of Ranked Society in the Coast Salish Region. In Reprint Proceedings Circumpacific Prehistory Conference, Seattle, Vol. 111, Part 2. Unpaginated.

Carlson, R. L. (1994). Trade and Exchange in Prehistoric British Columbia. In Baugh, T. G. and Ericson, J. E. (Eds.) Prehistoric Exchange Systems in North America. (pp. 307-364). New York: Plenum Press, New York

Clague, J. J., Harper, J.R., Hebda, R. J. and Howes, D. E. (1982). Late Quaternary Sea Levels and Crustal Movements, Coastal British Columbia. Canadian Journal of Earth Sciences 19, 597-618.

Clague, J. J. and James, T. S (2002). History and Isostatic Effects of the Last Ice Sheet in Southern British Columbia. Quaternary Science Reviews, 21, 71 - 87.

Clark, T. N. (2013). Rewriting Marpole: The Path to Cultural Complexity in the Gulf of Georgia. Mercury Series. 
Ottawa: University of Ottawa Press.

Close, A. (2006). Finding the People Who Flaked the Stone at English Camp (San Juan Island). Salt Lake City: University of Utah Press.

Conca, D. (2000). Archaeological Investigations at Site 45-CA-432: Re-evaluating Mid-Holocene Land Use on the Olympic Peninsula, Washington. Unpublished Master's Thesis. Western Washington University, Bellingham, Washington.

Conrey, R. M., Goodman-Elgar, M., Bettencourt, N., Seyfarth, A., Van Hoose, A., and Wolff, J. A.. (2014). Calibration of a portable X-ray fluoresence spectrometer in the analysis of archaeological samples using influence coefficients. Geochemistry, Exploration, Environment, Analysis. 14(3), 291.

Crabtree, D. (1972). An Introduction to Flintworking. Occasional Papers of the Idaho State University Museum, No. 28. Pocatello: Department of Anthropology, Idaho State University.

Darwent, J. (1996). The Prehistoric Use of Nephrite on the British Columbia Plateau. Unpublished Master's Thesis, Simon Fraser University. Burnaby, British Columbia.

Doelman, T., Torrence, R. Popov, V., Ionescu, M., Kluyev, N., Sleptsov, I., Pantyukhina, I., White, P. and Clements, N. (2008). Source Selectivity: An Assessment of Volcanic Glass Sources in the Southern Primorye Region, Far East Russia. Geoarchaeology, 23(2), 243-273.

Duer, D. and Turner, N. (Eds.) (2005). Keeping it Living: Traditions of Plant Use and Cultivation on the Northwest Coast of North America. Seattle: University of Washington Press.

Eis, S. and Craigdallie, D. (1980). The Gulf Islands of British Columbia: A Landscape Analysis . Canadian Forestry 
Service, Victoria: Pacific Forest Research Centre.

Fedje, D.W., Sumpter, I.D. and Southon, J. R. (2009). Sea-levels and Archaeology in the Gulf Islands National Park Reserve. Canadian Journal of Archaeology, 33(2), 234-253.

Graesch, A. (2007). Modeling Ground Slate Knife Production and Implications for the Study of Household Labor Contributions to Salmon Fishing on the Pacific Northwest Coast. Journal of Anthropological Archaeology, 26, 576606.

Green, A. J., van Vliet, L. J. P. and Kenney, E. A. (1989). Soils of the Gulf Islands of British Columbia vol. 3: Soils of Galiano, Valdes, Thetis, Kuper and Lesser Islands. Vancouver: British Columbia Soil Survey.

Grier, C. (2001). The Social Economy of a Northwest Coast Plankhouse. Unpublished Doctoral Dissertation. Arizona State University. Tempe, Arizona.

Grier, C. (2003). Dimensions of Regional Interaction in the Prehistoric Gulf of Georgia. In Matson, R. G., Coupland, G., and Mackie, Q. (Eds.) Emerging from the Mist. (pp. 170-188.) Vancouver: University of British Columbia Press.

Grier, C. (2006). Temporality in Northwest Coast Households. In Sobel, E. A., Trieu Gahr, D. A., and Ames, K. M. (Eds.) Household Archaeology on the Northwest Coast. (pp. 97-119.) Ann Arbor: International Monographs in Prehistory.

Grier, C. (2014). Landscape Construction, Ownership and Social Change in the Southern Gulf Islands of British Columbia. Canadian Journal of Archaeology, 38(1), 211-249.

Grier, C., and McLay, E. (2007). Archaeological Inventory of DgRv-006 Dionisio Point Provincial Park, Galiano 
Island. Report on file with the Archaeological Branch. Victoria [Heritage Permit 2007-144].

Grier, C., Dolan, P., Derr, K., and MacLay, E. (2009). Assessing Sea Level Change on the Southern British Columbia Coast using Archaeological Data from Cuspate Spits in the Gulf Islands. Canadian Journal of Archaeology, 33, 254-280.

Herbel, B. C., Olson, D.L., and Schwarzmiller, R. (2001). Artifact Descriptions. In R. Schalk, Kenady, S., Herbel, B., Schwarzmiller, R., Kopperl, R.E., Olson, D.L., and Breidenthal, M., (Eds.) Archaeological Testing at Cama Beach State Park. Cascadia Archaeology, Report Submitted on behalf of Washington State Parks and Recreation Commission, on file at the Department of Archaeology and Historic Preservation, Olympia, Washington.

Heiri, O., Lotter, A. F., and G. Lemcke, G. (2001). Loss on Ignition as a Method for Estimating Organic and Carbonate Content in Sediments: Reproducibility and CRmparability of results. Journal of Paleolimnology, 25, 101110.

Jaupert, C. and Tait, S. (2012). Dynamics of Differentiation in Magma Reservoirs. Journal of Geophysical Research, 100(B9), 17615-17636.

Johnson, D. M., Hooper, P. R. and Conrey, R. M.. (1999). XRF Analysis of Mocks and minerals for Major and Trace Elements on a Single Low Dilution Li-tetraborate Fused Bead. Advances in X-ray Analysis, 41, 843-867.

Johnstone, P. D. (2006). Geology of the Upper Cretaceous Nanaimo Group, Southernmost Gulf Islands and Adjacent Saanich Penninsula, Southwestern British Columbia. Unpublished Master's of Science Thesis, Department of Earth Sciences, Simon Fraser University, Burnaby, British Columbia.

Kenady, S. M., Saastamo, S. A., and Sprague, R. (1973). Miscellaneous San Juan Island Reports, 1970-1972. Issue 7 of University of Idaho anthropological research manuscript series. Moscow: University of Idaho, Department of 
Anthropology and Sociology .

Kim, J. and Grier, C. (2006). Beyond Affluent Foragers. In Grier, C., Kim, J. and Uchiyama, J. (Eds.) Beyond Affluent Foragers: Rethinking Hunter-Gatherer Complexity. (pp. 192-200.) Oxford: Oxbow Press.

King, A. R. (1950). Cattle Point, A Stratified Site in the Southern Northwest Coast Region. Society for American Archaeology Memoirs 7. Washington, D.C.: The Society for American Archaeology Press.

Kornbacher, K. D. (1989). Shell Midden Lithic Technology: An Investigation of Change at British Camp (45SJ24), San Juan Island. Unpublished Master's thesis, University of British Columbia. Vancouver, British Coumbia.

Kornbacher, K. D. (1992). Shell Midden Lithic Technology: Analysis of Stone Artifacts from British Camp, In Stein, J. K. (Ed.) Deciphering a Shell Midden. (pp. 163-191.) New York: Academic Press.

Kwarsick, K. C. (2010). Lithic Raw Material Procurement and Technological Organization of Olympic Peninsula Peoples. Unpublished Master's Thesis. Washington State University. Pullman, Washington.

Le Maitre, R. W. (Ed.), Streckeisen, A., Zanettin, B., Le Bas, M. J., Bonin, M. B., Bateman, M. P., Bellieni, M. G., Dudek, M. A., Efremova, M. S., Keller, M. J., Lamere, J., Sabine, P. A., Schmid, R., Sorensen, H. and Woolley, A. R.. (2002). Igneous Rocks: A Classification and Glossary of Terms, Recommendations of the International Union of Geological Sciences, Subcommission of the Systematics of Igneous Rocks. Cambridge: Cambridge University Press.

Lepofsky, D.,Schape, D. M., Graesch, A., Lenert, M., Ormerod, P., Carlson, K. C., Arnold, J. E., Blake, M., Moore, P., and Clague, J. J. (2009). Exploring Stó:lo-Coast Salish Interaction and Identity in Ancient Houses and Settlements in the Fraser Valley, British Columbia. American Antiquity, 74(4), 595-626. 
Matson, R. G. and Coupland, G. (1994). The Prehistory of the Northwest Coast. San Diego: Academic Press.

McKechnie, I. (2014). An Archaeology of Food and Settlement on the Northwest Coast.

Unpublished Doctoral Dissertation. University of British Columbia, Vancouver, British Columbia.

Mitchell, D. H. (1971a). Archaeology of the Gulf of Georgia Area: A Natural Region and its Culture Types. Syesis Supplement 1. Victoria: British Columbia Provincial Museum.

Mitchell, D. H. (1971b). The Dionisio Point Site and Gulf Island Culture History. Syesis, 4, 145-168.

Morgan, V. (Ed.) (1999). The SR-101 Sequim Bypass Archaeological Project: Mid- to Late-Holocene Occupations on the Northern Olympic Peninsula, Clallam County, Washington. Eastern Washington University Reports in Archaeology and History 100-108, Archaeological and Historical Services, Eastern Washington University, Cheney. Special Collection: F899.S495 S72 1999.

Morin, J. (2012). The Political Economy of Stone Celt Exchange in Pre-contact British Columbia: the Salish nephrite/jade industry. Unpublished Doctoral Dissertation. University of British Columbia. Vancouver, British Columbia.

Moss, M. L. (2011). Northwest Coast: Archaeology as Deep History. Washington, D.C.: The Society for American Archaeology Press.

Reimer, R. (2011). The Rocks are Forever: Lithics and Landscapes of Skwxwú7mesh Uxwumixw. Unpublished Doctoral Dissertation. McMaster University. Hamilton, Ontario.

Reimer, R. and Hamilton, T. (2015). Implications between Technological Organization and Portable X-Ray Fluorescence Analysis on Lithic Material Use at Two Rockshelter Sites on the southern Northwest Coast. In Adams, 
R. and Ozbun, T. (Eds.) Toolstone Geography. SFU Archaeology Press. Burnaby, British Columbia.

Rorabaugh, A. N., and McNabb, C. Y. (2014). A Geospatial Analysis of Toolstone Acquisition and Use: A Preliminary Investigation of Material Quality and Access Over 4,000 years in the Salish Sea. Canadian Journal of Archaeology, 38(2), 371-393.

Schaepe, D. (2009). Pre-colonial Stó:lō-Coast Salish community organization: An archaeological study.

Unpublished Doctoral Dissertation. University of British Columbia. Vancouver, British Columbia.

Schalk, R. (1988). The Evolution and Diversification of Native Land Use Systems on the Olympic Peninsula: A Research Design. Report prepared for National Park Service Pacific Northwest Region for Contract No. CX-900-4E075 by the Institute for Environmental Studies, University of Washington. Report on file at Olympic National Park.

Skinner, C. (2009). Lost and Found in British Columbia: Central Coast A. Northwest Research Obsidian Studies Lab. Electronic Document Accessed 10/20/12.

$<$ http://sourcecatalog.blogspot.com/2009/08/lost-and-found-in-british-columbia.html>

Sobel, E. (2006). Household Prestige and Exchange on the Northwest Coast: A Case Study of the Lower Columbia River Valley. In Sobel, E., Trieu Gahr, A. and Ames, K. (Eds.) Household Archaeology on the Northwest Coast. (pp. 159-199.) Ann Arbor: International Monographs in Prehistory.

Stein, J. K. (1992). Organic Matter in Archaeological Contexts. In Holliday, V. T. (Ed.) Soils, Landscape Evolution, and Human Occupation, (pp. 193 - 216.) Washington, D. C.: Smithsonian Institution Press.

Stein, J. K. (2000). Exploring Coast Salish Prehistory, The Archaeology of San Juan Island. Burke Museum Monographs. Seattle: University of Washington Press. 
Stewart, H. (1996). Stone, Bone, Antler, and Shell- Artifacts of the Northwest Coast. Seattle: University of Washington Press.

Sullivan, A. P. and Rozen, K. C. (1985). Debitage Analysis and Archaeological Interpretation. American Antiquity, $50,755-779$.

Taylor, A. K. (2012). Creating and Transcending Territorial Boundaries in Late Holocene Pacific Coast Communities. Unpublished Doctoral Disseration. University of Washington. Seattle, Washington.

Taylor, A. K. and Skinner, C. (2010). A Geochemical Analysis of San Juan Islands Fine-Grained Volcanic Toolstone. Paper presented at the Northwest Anthropological Conference. Ellensburg, WA.

Terry, K., Andrefsky, W., and Konstantinov, M. K. (2009). Raw Material Durability, Function, and Retouch in the Upper Paleolithic of the Transbaikal Region, Siberia. In Adams, B. and Blades, B. S. (Eds.). Lithic Materials and Paleolithic Societies. (pp. 256-269.) New York: Wiley-Blackwell

Thom, B. (1995). The Dead and the Living: Burial Mounds and Cairns and the Development of Social Classes in the Gulf of Georgia Region. Unpublished Master's Thesis. University of British Columbia. Vancouver, British Columbia.

Wessen, G. (1993). An Overview of Archaeological Activities Conducted by Western Heritage, Inc. in the Lake Cushman Project Area, 1988-1991. Report Prepared for Tacoma Public Utilities, Tacoma, Washington by Wessen and Associates, Seattle, Washington.

Whittaker, J. C. (1994). Flintknapping: Making and Understanding Stone Tools. Austin: University of Texas Press. 
Wilson, L. (2007). Understanding Prehistoric Lithic Raw Material Selection: Application of a Gravity Model. Journal of Archaeological Method and Theory, 14, 388-411. 